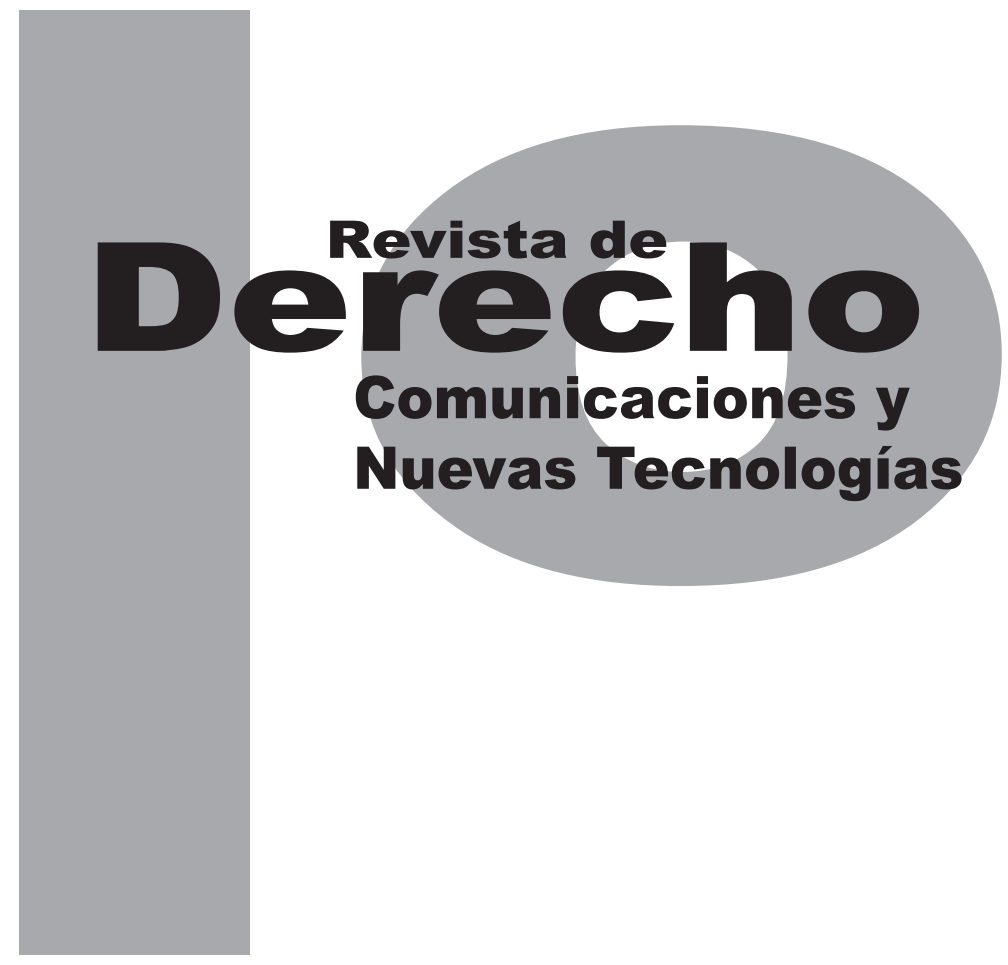

\title{
EL RÉGIMEN JURÍDICO APLICABLE A LAS ACTIVIDADES EN LA LUNA Y OTROS CUERPOS CELESTES
}

\author{
Carolina Araujo Chovil \\ ARMANDO GUIO ESPAÑOL
}

Universidad de los Andes

Facultad de Derecho

Revista de Derecho, Comunicaciones y Nuevas Tecnologías

No. 10, Julio - Diciembre de 2013. ISSN 1909-7786 


\title{
El régimen jurídico aplicable a las actividades en la luna y otros cuerpos celestes*
}

\author{
Carolina Araujo Chovil \\ Armando Guio Español ${ }^{* *}$
}

\section{RESUMEN}

La Luna es un cuerpo celeste de enorme importancia en la cultura mundial que se ha considerado como patrimonio universal. Sin embargo, este concepto se ha puesto en duda debido a que las naciones que han tenido la posibilidad de realizar exploraciones en este satélite natural han planteado su posible apropiación. Esto generó una preocupación dentro de varios países, llevando a que en 1979 se presentara en el seno de las Naciones Unidas la redacción del Acuerdo que debe regir las actividades de los Estados en la Luna. Dentro esta normatividad se destaca el artículo 11ro, en el que se estableció un innovador régimen que garantizaba a todos los países el acceso equitativo a los recursos que se hallen en la Luna. En este trabajo se explica el contexto y los principios que inspi-
The Moon is a celestial body of huge importance in human culture and has been considered as a world heritage. However, this concept has been in doubt due to the fact that the nations that had the possibility to explore this natural satellite have considered ways of appropriating it. This concern led to the creation in 1979 within the United Nations of a juridical regime that regulated the activities of States in the Moon. Article 11, of these rules established an innovative regime that guarantied the equitable access of all countries to the resources allocated in the Moon. This paper explains the context and principles that inspired this agreement, as well as its qualities and defects and describes some proposals in order to improve it.

Cómo citar este artículo: Araujo Chovil, C., y Guio Español, A. (Diciembre, 2013). El régimen jurídico que rige las actividades en la luna y otros cuerpos celestes. Revista de Derecho, Comunicaciones y Nuevas Tecnologías, 10.

** Estudiantes de décimo semestre de la Facultad de Derecho de la Universidad de los Andes. Correos: c.araujo955@uniandes.edu.co y a.guio139@ uniandes.edu.co 
raron este acuerdo, se expondrán sus cualidades y defectos y se sugieren algunas propuestas para mejorarlo

Palabras clave: Derecho del Espacio Ultraterrestre, No apropiación del Espacio, Explotación de recursos naturales, Patrimonio Universal, Luna y cuerpos celestes, Tecnología Espacial, Acuerdo de 1979.
KEYWORDS: Space Law, space appropriation, Natural resources exploitation, World heritage, Moon and celestial bodies, Space Technology, 1979 Agreement 


\section{SUMARIO}

Introducción - I. IMPORTANCIA CULTURAL E HISTÓRICA DE LA LUNA Y SU EXPLORACIÓN - A. Los cuerpos celestes en la antigüedad y el mito alrededor de la Luna: el rol de la luna en la construcción del imaginario del hombre - B. Contexto histórico que rodea la exploración lunar y el Derecho de Conquista - II. PRINCIPIOS QUE RIGEN LA EXPLORACIÓN ESPACIAL - A. Tratado del Espacio Ultraterrestre: normativa básica aplicable a la Luna y otros cuerpos celestes - III. ACUERDO QUE DEBE REGIR LAS ACTIVIDADES DE LOS ESTADOS EN LA LUNA Y OTROS CUERPOS CELESTES DE 1979 - A. Estatus del Tratado - B. El régimen de apropiación de los elementos y recursos descubiertos en la Luna y el concepto de "patrimonio común de la humanidad" - V. EL ARTÍCULO 11 DEL TRATADO DE LA LUNA: LOS RECURSOS NATURALES DE ESTE CUERPO COMO PATRIMONIO DE LA HUMANIDAD - A. El debate sobre el significado del término patrimonio común de la humanidad como una manifestación de la tensión entre países desarrollados y en vía de desarrollo-B. El significado de la expresión patrimonio común de la humanidad está dado por las disposiciones mismas del Tratado de la Luna, su objeto y fin, y está circunscrito al desarrollo de un nuevo régimen internacional que rija la explotación de recursos naturales - V. PROBLEMAS ESPECÍFICOS RELATIVOS AL TRATADO DE LA LUNA - A. ¿Cuál es la fuerza vinculante del Tratado de la Luna? - B. El Acuerdo que debe regir las actividades de los Estados en la Luna y otros cuerpos celestes: ¿un inminente fracaso? Análisis de las razones por las cuales la mayoría de Estados no han ratificado el Tratado de la Luna - C. ¿El Acuerdo también prohíbe la propiedad privada sobre la Luna u otros cuerpos celestes? - 1. Casos de reivindicaciones de soberanía por parte de particulares y compañías privadas - 2. La imposibilidad de establecer propiedad privada en la luna y otros cuerpos celestes - V. LA REGULACIÓN SOBRE LA LUNAY OTROS CUERPOS CELESTES EN EL FUTURO ¿QUÉ SE ESPERA? - A. Propuestas para un futuro régimen jurídico internacional que rija la explotación de los recursos naturales del espacio ultraterrestre - VII. CONCLUSIONES - Bibliografía 
Introducción

Uno de los temas que mayor interés ha generado en el hombre a lo largo de la historia es entender la composición y características de los diversos cuerpos celestes que lo rodean. Desde épocas arcaicas el hombre ha tratado de darle una explicación a la presencia de aquellos objetos que logra observar en el espacio y lo ha asociado con todo tipo de mitos e historias, tal como lo hicieran los griegos o romanos en su momento.

Hace pocas décadas, la humanidad pudo cumplir el sueño de ponerse en contacto con estos cuerpos celestes y lo hizo a través del desarrollo de la tecnología espacial, que ha permitido la llegada de seres humanos a algunos cuerpos celestes como la Luna y el reconocimiento de muchos otros gracias a los satélites que se han enviado al espacio ultraterrestre. Este desarrollo ha permitido conocer las características de diversos cuerpos celestes y su estructura, para así determinar si pueden ser terrenos explorables o no. Sin embargo, y como suele suceder en todo proyecto que emprende la humanidad, la ausencia definitiva de normas que regulen esta exploración puede conllevar grandes problemáticas, ya que es un tema que se puede prestar para todo tipo de discusiones e inclusive conflictos internacionales debido, especialmente, a la desigualdad que existe en el desarrollo tecnológico espacial entre los países. De esta forma, el presente artículo busca, no sólo entender la regulación actual que se ha generado frente a este fenómeno, sino que intentará evidenciar si la normativa existente es suficiente para los nuevos retos y exploraciones que se están presentando y si resulta entonces necesaria una propuesta de desarrollo frente a este tema que atienda no solo a factores técnicos y políticos, sino que tenga también un sustento jurídico acorde al desarrollo y los principios que rigen el derecho espacial.

Para lograr este objetivo se identificaron tres problemas jurídicos principales a partir de los cuales se evidenciarán los mayores desafíos que experimenta la actual regulación frente a la exploración y utilización de la Luna y otros cuerpos celestes. El primero de ellos plantea el aparente fracaso del tratado internacional que regula las actividades de los Estados en la Luna y otros cuerpos celestes, ligado al hecho que son muy pocos los Estados que son arte del mismo y su no ratificación por parte de los países que llevan a cabo la mayor parte de la actividad espacial. El segundo problema radica en las reivindicaciones de soberanía sobre la Luna por parte de compañías privadas y particulares, quienes aseguran tener derechos de propiedad sobre la misma. El último problema jurídico identificado, tal vez el más importante, recae en la pregunta sobre: ¿cuál debe ser el tratamiento y regulación aplicable a los recursos naturales del espacio ultraterrestre y su calificación como "patrimonio común de la humanidad" en el Tratado de la Luna? Finalmente se plantearán propuestas para construir un nuevo régimen internacional que rija la explotación y utilización de los recursos naturales extraídos de la Luna y otros cuerpos celestes con el fin de que pueda ser tenido en cuenta para un futuro tratado internacional al respecto. Vale la pena agregar 
que el presente trabajo se enfocará en analizar la Luna y su régimen jurídico, sin perjuicio que todo lo dicho respecto de la misma pueda ser aplicable a otros cuerpos celestes.

\section{IMPORTANCIA CULTURAL E HISTÓRICA DE LA LUNA Y SU EXPLORACIÓN}

\section{A. Los cuerpos celestes en la antigüedad y el mito alrededor de la Luna: el rol de la luna en la construcción del imaginario del hombre.}

La Luna ha sido objeto de fascinación, intriga y todo tipo de historias, pero ante todo ha sido un actor principal en el imaginario que caracteriza al ser humano. Hoy encontramos una humanidad dividida en diversas culturas, religiones y tradiciones, sin olvidar la separación que se ha generado entre lo que se cataloga como "oriente" y "occidente"; sin embargo, el tema de la Luna es abordado por muchas de estas culturas o bloques a lo largo de la historia y cualquier ser humano, sin importar su procedencia, se ha sentido intrigado por esta.

Una de las primeras formas que tuvo el hombre para explicar al existencia de la Luna fue a través de la religión. Para los Incas, la luna era una mujer que protegía a los animales y seres de la naturaleza, por lo que se le denominaba Mama Quilla (Cobo, 1653, p. 29-32). Los egipcios la denominaron Jonsu y era la diosa que protegía a los humanos de los malos espíritus y al mismo tiempo que cuidaba de la reproduc- ción y la fertilidad. En la Cultura Griega se denominaba Selene y se le asociaba con el amor y la belleza; mientras que en Roma sería conocida como la diosa Luna, tal como se le denomina actualmente (Grimal, 1986, p. 415). Los chinos tampoco fueron ajenos a esta mitología y denominaron a la Luna como Chang'e, aquella que brindaba belleza y juventud a quienes la adoraban. El programa chino de exploración en la Luna recibe el nombre de esta diosa (Laxman, 2012). Estos son algunos ejemplos que demuestran la importancia y diversidad mitológica que ha tenido este cuerpo celeste a lo largo de múltiples y distintas culturas. Una vez se fueron constituyendo religiones monoteístas, como el judaísmo, el catolicismo o el islamismo, la Luna perdió ese carácter divino, pero no por eso su trascendencia en la cultura y esto, gracias a la literatura universal, que la tomo como uno de sus más importantes objetos de inspiración.

Aunque son innumerables los textos que abarcan la Luna y figuras literarias asociadas con la misma, tales como el hombre lobo, existe una en especial: la obra de Julio Verne Alrededor de la Luna (Autour de la Lune). En esta obra se describe uno de los principales sueños de la sociedad científica europea de la época: llegar a la Luna. La obra relata la historia de tres exploradores que son enviados en un objeto parecido a una bala a conquistar el espacio. La obra de Verne ha resultado de un interés enorme no solo por la forma como expresa la fascinación del hombre con la idea de llegar a la Luna, sino que describe los últimos avances científicos en el tema, por lo que el texto se ha considerado como premonitorio. Son interesantes las coincidencias, no sólo 
en la existencia de tres primeros exploradores, como serían los tres miembros del Apolo 11, la salida del cohete desde un lugar costero o que en su vuelta a la tierra llegaran al mar, como en realidad sucedería (Crovisier, 2012).

\section{B. Contexto histórico que rodea la explo- ración lunar y el derecho de conquista}

En el momento en el que Neil Armstrong se convierte en el primer ser humano en pisar la Luna, en la Tierra se vivía una situación histórica única y esta exploración no sería ajena a este momento. En el año 1969, la humanidad se dividía en dos posiciones ideológicas completamente opuestas, el capitalismo y el comunismo, el primero representado por los Estados Unidos de América y el segundo por la Unión de Repúblicas Socialistas Soviéticas. A pesar de las constantes tensiones existentes entre ambos países, en el que surgieron distintas amenazas y provocaciones, nunca se produjo un conflicto bélico directo entre las dos potencias, por lo que dicho periodo se denominó como la Guerra Fría (Lozano, 2007).

La exploración espacial no fue ajena a este contexto de enfrentamiento ideológico y militare. Varios autores afirman que el desarrollo espacial se usó estratégicamente como medio de intimidación y también para desarrollar la tecnología, no solo en provecho de la exploración espacial, sino como estrategia para una futura exploración militar (Artola, 2009). Aunque no hay completa seguridad de estas teorías, lo que sí es cierto es que la exploración espacial se convirtió en una carrera espacial donde se produjeron diferentes metas y donde, sorprendentemente, la Luna tendría una importancia trascendental.

En primer lugar, el objetivo era llevar un objeto al espacio y que el mismo fuera rastreable y controlable. La primera victoria llegaría para los soviéticos con el Sputnik en 1956, que sería el primer satélite artificial en orbitar la tierra. Esto incrementó las preocupaciones de los científicos norteamericanos, quienes sabían que debían demostrar que también contaban con los recursos e intelecto para lograr lo mismo que sus pares soviéticos (Artola, 2009). Sin embargo, mientras los norteamericanos fallaban en sus intentos de poner un objeto en el espacio, los soviéticos ya ponían al primer ser viviente en el espacio, con la perra Laika, que se convertiría en un ícono de este enfrentamiento por el espacio. Cuando por fin los norteamericanos lograron localizar objetos en el espacio, los soviéticos fueron mucho más allá en esta competencia e hicieron que el coronel Yuri Gagarin lograra salir al espacio ultraterrestre y orbitar la Tierra, convirtiéndose en el primer ser humano en el espacio. La hazaña lograda con el viaje de Gagarin y su llegada sano y salvo a la tierra, significó un enorme triunfo para los soviéticos, quienes sabían que sus exploraciones superaban por mucho las de sus competidores (Martos, 2009). Los norteamericanos sabían que sólo un hecho podría superar todo lo ya hecho por los soviéticos y esto no era otra cosa que la llegada a la Luna. Llegar a la Luna significaba conquistar uno de los sueños que había tenido la humanidad a lo largo de su historia, descubrirla, caminar sobre ella, ver cómo era, resultaba algo asombro- 
so y trascendental. Los norteamericanos eran conscientes de la importancia de este cuerpo celeste y dirigieron todos sus planes hacia este fin. Se estima que el gobierno Kennedy brindó recursos ilimitados a la NASA para desarrollar el proyecto Lunar (The Decision to go to the Moon, 2012). Por lo tanto, los norteamericanos no se preocuparon por llevar una persona solo hasta el espacio, sino que ella debía llegar hasta la Luna.

Para este plan fueron elegidos tres pilotos de la fuerza aérea norteamericana: Neil Armstrong, Buzz Aldrin y Michael Collins. La misión de estos militares sería llegar a la Luna, explorar parte de la misma y llegar sanos y salvos a la tierra. Fue así que los primeros norteamericanos que salieron al espacio el 16 de Julio de 1969, lograrían el principal sueño de la humanidad y darían un adelanto en la carrera espacial, imposible de superar. Luego de 5 días de viaje, finalmente, el 21 de julio de 1969 Neil Armstrong, junto con Aldrin, serían los primeros seres humanos en alunizar y el primero daría aquel paso en la Luna que quedará marcado por siempre en la historia de la humanidad. La importancia de dicha llegada fue inmensa. Los norteamericanos habían logrado con esta campaña sacudirse la presión de los adelantos soviéticos y se considera que solo con este hecho superaron todo lo hecho anteriormente. La victoria americana con la llegada a la Luna, nos demuestra la importancia que tiene este cuerpo celeste para la humanidad y el impacto que tuvo la llegada al mismo. Las connotaciones políticas de esta exploración son incalculables y es que la Luna se ha convertido en el destino de toda nación que desea demostrar su poderío y desarrollo tecnológico. Por ejemplo, hoy en día la China está muy cerca de enviar un hombre a la superficie Lunar, con el fin, no solo de demostrar su desarrollo científico, sino con fines políticos propagandísticos (How China may be the next to land on the moon, 2012).

Sin embargo, este descubrimiento supuso otros desafíos, especialmente determinar si los Estados Unidos se apropiarían de la misma, si la Luna podía tener un dueño o seguiría siendo patrimonio de la humanidad como lo venía siendo desde antes. Es importante recordar que desde el descubrimiento de América cinco siglos antes, el hombre no se enfrentaba a un descubrimiento tan importante. En el caso del descubrimiento americano, lo que se presentó fue ante todo una conquista del territorio descubierto y para esto se impuso un sistema político y jurídico que sustentara este proceso expansionista, generando graves consecuencias como fue la violencia, el asesinato de millones de indígenas y la destrucción y olvido de milenarias culturas ancestrales.

Teniendo en cuenta las experiencias del pasado, la comunidad internacional acordó que al momento de crear un régimen jurídico sobre el espacio se debía superar la teoría del derecho de conquista para así evitar seguir con modelos violentos y basados en la guerra. Esta superación era posible, según sus defensores, pues ya existían entidades de cooperación internacional como las Naciones Unidas y los discursos imperialistas cada vez tenían menos aceptación, especialmente con los procesos de independencia que se vivieron en América y África en los últimos dos siglos. 
Increíblemente, un acuerdo que regulará las actividades de los Estados sobre la Luna se presentó hasta 1979, diez años después de la llegada del Apolo 11 a este cuerpo celeste. Esto se debe a que el legislador internacional consideró prioritario regular antes otros temas vitales para la actividad espacial que se estaban llevando a cabo en la época. Antes del Acuerdo de la Luna se aprobaron otros tratados en materia espacial tales como: el Tratado sobre los principios de la gobernanza de las actividades de las naciones en la exploración y uso del espacio exterior, incluyendo a la Luna u otros cuerpos celestes (1967), el Tratado sobre salvamento y devolución de astronautas (1967), el Tratado sobre responsabilidad por daños causados por objetos lanzados al espacio (1972 )y el Convenio sobre registro de objetos y propiedad de los objetos espaciales (1974). Es importante conocer el desarrollo previo, ya que el Acuerdo de la Luna se valdrá de los acuerdos y tratados anteriores al mismo para generar un régimen legal sobre los cuerpos celestes (United Nations Committee on the Peaceful Uses of Outer Space, COPUOS, 2008). Es así como surgió una regulación internacional sobre el espacio y debido a su importancia, una normatividad específica relativa a la Luna, la cual pasaremos a analizar.

\section{PRINCIPIOS Y TRATADOS QUE RI- GEN LA EXPLORACIÓN ESPACIAL}

\section{A. Tratado del Espacio Ultraterrestre: normativa básica aplicable a la Luna y otros cuerpos celestes}

Con el desarrollo de la actividad espacial y la inminente llegada del hombre a la Luna, la comunidad internacional identificó la necesidad de producir un instrumento internacional jurídicamente vinculante para regular la recién surgida actividad espacial y evitar su incursión en las dinámicas de la Guerra Fría. Así pues, en el seno de la Asamblea General de las Naciones Unidas el 19 de diciembre de 1966 mediante Resolución 2222 (XXI) se aprobó el Tratado sobre los principios que deben regir las actividades de los Estados en la exploración y utilización del espacio ultraterrestre, incluso la Luna y otros cuerpos celestes ${ }^{1}$, mejor conocido como el Tratado sobre el espacio ultraterrestre o Tratado del 67, el cual entra en vigor el 10 de octubre de 1967 y hoy cuenta con 101 Estados Partes (Treaty Database: Treaty signatures, 2013. Cursivas nuestras). Dicho tratado habría de ser considerado en adelante como la Constitución del espacio, en la medida que establece unos principios guías que habrían de constituir las bases para la actual regulación jurídica internacional sobre las actividades espaciales. Su importancia radica en que: "todos los tratados espaciales de Ias Naciones Unidas hacen referencia al tratado

El antecedente jurídico inmediatamente anterior del tratado del espacio ultraterrestre es la Resolución 1962 (XVIII) de 13 de diciembre de 1963 conocida como la Declaración de principios de 1963 
del espacio ultraterrestre como su fundamento y elaboran más a fondo algunos de sus principios" (Kopal, 2009, p.8), incluido el Acuerdo que debe regir las actividades de los Estados en la Luna y otros cuerpos celestes de 1979, relativo a la reglamentación particular aplicable a la Luna y otros cuerpos celestes.

Hay que tener en cuenta que todas las disposiciones y principios señalados en el Tratado del 67 no sólo son aplicables al espacio ultraterrestre sino de manera simultánea aplican a la Luna y otros cuerpos celestes, razón por la cual, es posible afirmar que la primera regulación aplicable a Luna y otros cuerpos celestes es el Tratado del Espacio Ultraterrestre. Vale la pena resaltar que algunos de los principios enunciados en el tratado del 67 no solo son los principios rectores de toda actividad espacial, sino que además han pasado a ser concebidos como Derecho Internacional Consuetudinario derivado de un tratado $y$, por ende, jurídicamente vinculantes ${ }^{2}$. Entre estos principios está el principio de no apropiación del espacio ultraterrestre incluida la luna y otros cuerpos celestes por reivindicaciones de soberanía, uso u ocupación, ni de ninguna otra manera; el principio de uso pacífico del espacio ultraterrestre incluida la Luna y otros cuerpos celestes; el principio de no utilización de armas nucleares en el espacio ultraterrestre, la luna y otros cuerpos celestes y el uso de la energía nuclear con fines pacíficos exclusivamente; el principio que establece la libertad de exploración y utilización del espacio ultraterrestre, la luna y

2 Según los autores Lyall y Larsen (2009) los artículos 1-4 del tratado del espacio ultraterrestre han pasado a ser considerados normas de Derecho Internacional Consuetudinario. (p.175-199). otros cuerpos celestes en provecho e interés de todos los países sea cual fuere su grado de desarrollo económico y científico pues incumben a toda la humanidad y el principio de cooperación internacional.

Teniendo en cuenta lo anterior, incluso los Estados que aún no han ratificado el Tratado del 67 deben cumplir con tales principios en virtud de que han pasado a ser concebidos como costumbre internacional en los términos del artículo $38^{\text {vo }}$ de la Convención de Viena sobre el derecho de los tratados (1969). ${ }^{3}$

\section{ACUERDO QUE DEBE REGIR LAS ACTIVIDADES DE LOS ESTADOS EN LA LUNA Y OTROS CUERPOS CELESTES DE 1979}

La normativa internacional específica aplicable en lo relativo a las actividades en la Luna y otros cuerpos celestes es el Acuerdo que debe regir las actividades de los Estados en la Luna y otros cuerpos celestes de 1979 y por ende será objeto de un análisis detallado en este artículo.

Tal y como lo establecen los profesores Diederiks-Verschoor y Kopal en su obra An Introduction To Space Law los dos principales motivos para que en 1979 se realizara un tratado sobre la Luna fueron: i )la necesidad de hacer de la

3 El citado artículo dispone lo siguiente: "normas de un tratado que lleguen a ser obligatorias para terceros Estados en virtud de una costumbre internacional. Lo dispuesto en los artículos 34 a 37 no impedirá que una norma enunciada en un tratado llegue a ser obligatoria para un tercer Estado como norma consuetudinaria de derecho internacional reconocida como tal" (Convención de Viena sobre el derecho de los tratados, 1969, Art. 38). 
Luna un objeto libre de conflictos entre las potencias espaciales y ii )el deber de regular la extracción y uso de materiales que se hallaran en la Luna (Verschoor y Kopal, 2008, p. 48). Esta última la explica Ferrer, quien establece que la Subcomisión Jurídica de la Comisión sobre la Utilización del Espacio Ultraterrestre con Fines Pacíficos de las Naciones Unidas, al momento de plantear el tema de un tratado sobre la Luna, debía tener en cuenta los siguientes criterios:

i.) Es necesario dictar un estatuto propio para los recursos naturales del espacio ultraterrestre, la Luna y los cuerpos celestes ii.) Conforme al principio establecido en el art. 1 de dicho Tratado, la utilización deberá hacerse en provecho e interés de todos los países, sea cual fuere el grado de su desarrollo económico y científico e incumbe a toda la humanidad. De ello puede derivarse que los recursos naturales del espacio ultraterrestre, incluso la Luna y los cuerpos celestes, constituyen patrimonio común de la humanidad. iii.) Todo material originario de la Luna y los cuerpos celestes, constituyen el patrimonio de la Humanidad" (1976, p. 214-215).

Muchas de estas recomendaciones llegarían a ser tenidas en cuenta en una $u$ otra medida. $A$ continuación se pasará a explicar cuáles fueron los principales aportes de este Acuerdo.

En un principio se quiso que el acuerdo solo regulara la exploración en la Luna, sin embargo, hubo varios críticos a esta medida debido a que se considerada como un desperdicio de esfuerzo y recursos limitar la regulación a la Luna, por lo que se estableció que aunque este satélite natural sería el centro del acuerdo, el mismo haría referencia a otros cuerpos celestes, especial- mente aquellos presentes en el sistema solar y que solían ser los de más fácil acceso para la actividad exploratoria del hombre (Ferrer, 1976, p. 214). Esta regulación no impide que en un futuro se cree un régimen jurídico especial frente a algún cuerpo celeste, el cual aplicaría por el concepto de lex specialis derogat lex generali (Úbedad, 2011, p. 164). Así mismo, el artículo I del acuerdo en su numeral 3 también señala que en su campo de aplicación no contempla la entrada de cuerpos celestes a la superficie terrestre por medios naturales y propios de la naturaleza, tales como los asteroides y meteoritos (Verschoor y Kopal, 2008, p. 48-49).

Uno de los problemas del tratado que ha sido criticado en mayor medida es que a diferencia de otros tratados que componen el Corpus luris Espacialis, el Acuerdo sobre la Luna no contiene definiciones esenciales, como por ejemplo la de cuerpos celestes, por lo que genera dificultades en el momento de determinar el rango de aplicación de esta norma. Como lo recuerda el profesor Aldo Armando Cocca: "cabe aquí señalar que ni el espacio ultraterrestre, ni la Luna, ni los restantes cuerpos celestes cuentan al presente con un concepto jurídico. Se les dio un estatuto legal, más se carece de una definición jurídica" (Cocca, 1970, p. 662-663). El mismo autor señala que uno de los principales intentos por brindar definiciones en este campo lo constituyó el Primer Coloquio sobre los progresos en la exploración cósmica y sus consecuencias para la humanidad de 1966, que se celebró en Buenos Aires; lastimosamente esta propuesta no tuvo una buena acogida. 
Ahora bien, se ha establecido que una de las razones de la formación de este tratado fue evitar conflictos alrededor del régimen que debía regir la exploración lunar. Muchos de los principios generales desarrollados en el Tratado del 67 ya regulaban este tema con principios como el de no apropiación y de cooperación, sin embargo, el tratado de la Luna lo que quiso en un primer momento fue reafirmar estos principios y aplicarlos a este caso en concreto. Así, el artículo IX, parágrafo 2 del tratado señala la imposibilidad de apropiación de la Luna, ni siquiera por medio de su uso u ocupación. Siguiendo esta línea el tratado señala que la exploración Lunar debe presentarse sin discriminación, con base en la igualdad y de acuerdo a las normas de Derecho Internacional y con los tratados internacionales relativos al tema, unido a que la Luna se debe empezar a considerar como patrimonio y legado común a toda la humanidad. El tratado igualmente invita a tener en consideración la situación de los países en vía de desarrollo, al igual que se pide no desconocer los esfuerzos especiales de los países que más han contribuido directa o indirectamente en la exploración de la Luna. ${ }^{4}$

\section{A. Estatus del Tratado}

El Acuerdo que debe regir las actividades de los Estados en la Luna y otros cuerpos celestes (en adelante, Tratado de la Luna) fue aprobado por la Asamblea General de las Naciones Unidas el 5 de diciembre de 1979 por medio de la Reso-

$4 \quad$ Todo lo anterior se extrae del articulado del Acuerdo que debe regir las actividades de los Estados en la luna y otros cuerpos celestes. Resolución 3235 de la Asamblea General de la ONU de 1979.
Iución 34/68. Estuvo abierto a la firma desde el 18 de diciembre de 1979 (United Nations Treaty Collection, 2013) y entró en vigor el 11 de Julio de 1984, 30 días después de que el quinto instrumento de ratificación fuera depositado en Austria.

Hoy, 2013, son Parte del Acuerdo quince Esta$\operatorname{dos}^{5}$ y cuatro más han manifestado su intención de ratificar a través de su firma United Nations Treaty Collection 2013). Los últimos dos Estados en adherirse o ratificar el Tratado fueron Turquía, el 29 de febrero de 2012 y Arabia Saudita, el 18 de Julio de 2012 United Nations Treaty CoIlection, 2013).

Es de notar que ninguno de los países con programas espaciales vigentes son Partes del Tratado de la Luna: Estados Unidos, Rusia y China, las mayores potencias en términos espaciales y quienes desarrollan la mayor parte de la actividad espacial hoy día, no son Partes del mismo. De hecho, según señala Thomas Gangale, "ningún Estado de lanzamiento ${ }^{6}$ ha ratificado el tratado de la Luna y solamente dos estados de lanzamiento, Francia e India, Io han firmado" (2009, p. 84. Cursivas nuestras).
5 Son Partes del Tratado de la Luna: Australia, Austria, Bélgica, Chile, Kazakhstán, Líbano, México, Marruecos, los Países Bajos, Pakistán , Perú, Filipinas, Arabia saudita, Turquía y Uruguay.

El Artículo $1^{\text {ro }}$ literal c del Convenio sobre la responsabilidad internacional por daños causados por objetos espaciales señala que se entiende por "estado de lanzamiento": un Estado que lance o promueva el lanzamiento de un objeto espacial; ii )Un Estado desde cuyo territorio o desde cuyas instalaciones se lance un objeto espacial. 


\section{B. El régimen de apropiación de los ele- mentos y recursos descubiertos en la Luna y el concepto de "patrimonio co- mún de la humanidad"}

Para la doctrina experta en Derecho Espacial este punto es el que representa mayor novedad e interés del Acuerdo sobre la Luna y otros cuerpos celestes. Este punto se encuentra desarrollado en el artículo XI del acuerdo, el cual debe ser analizado de manera detallada para entender su importancia. Este artículo puede dividirse en dos elementos principales, en primer lugar se reconfirmará el principio de no apropiación, ahora en aplicación a los cuerpos celestes entre ellos la Luna y en segundo lugar se determina un régimen de uso de los elementos descubiertos en dichos cuerpos. La primera parte ya ha sido desarrollada y se ha establecido que en este punto se siguen los lineamientos del Tratado del 67 sobre el tema. En este punto solo vale la pena establecer que el acuerdo señala que la propiedad de la Luna o de otros cuerpos no podrá ser alegada por lo que se considera como el "emplazamiento de personal, vehículos espaciales, equipo, material, estaciones e instalaciones sobre o bajo la superficie de la Luna, incluidas las estructuras unidas a su superficie o la Subsuperficie" (Verschoor y Kopal, 2008, p. 49$50)$. Esto significa que inclusive la construcción e incorporación de edificaciones en la superficie de estos cuerpos no podrá generar derechos de propiedad y significa un solo derecho de uso, mas no permite la apropiación del mismo.

La segunda parte del artículo XI resulta ser la más interesante y novedosa de todas. La mis- ma surge debido a que después de las primeras exploraciones a la Luna, se empezaron a hallar elementos químicos con propiedades interesantes y que podían significar una ventaja considerable en su aplicación industrial y tecnológica. Se afirma que "entre 1969 y 1974, la NASA recogió aproximadamente 2.200 piedras Lunares" (Nasa reconoce que perdió piedras lunares y otras muestras del espacio, 9 de diciembre de 2011), las cuales han servido para toda clase de investigaciones. Esta actividad empezó a ser cuestionada por otras naciones, en especial el acceso a la información derivada de dicha actividad. Como producto de este fenómeno el artículo XI del Acuerdo, en su segunda parte, fijará los siguientes parámetros:

Los Estados Partes en el presente Acuerdo se comprometen a establecer un régimen internacional, incluidos los procedimientos apropiados, que rija la explotación de los recursos naturales de la Luna, cuando esa explotación esté a punto de llegar a ser viable. (Acuerdo que debe regir las actividades de los Estados en la Luna y otros cuerpos celestes, 1979, Art. XI).

Así, se busca la creación de un régimen jurídico de control a la explotación de materiales de la Luna, el cual genera como primera obligación el deber de informar a la comunidad internacional sobre los recursos naturales que se descubran. Esta normativa que regule el hallazgo de materiales y recursos seguirá los siguientes parámetros en su formación, según el artículo XI numeral 7:

a.) El desarrollo ordenado y seguro de los recursos naturales de la Luna; 
b.) La ordenación racional de esos recursos;

c.) La ampliación de las oportunidades para el uso de esos recursos;

d.) Una participación equitativa de todos los Estados Partes en los beneficios obtenidos de esos recursos, teniéndose especialmente en cuenta los intereses y necesidades de los países en desarrollo, así como los esfuerzos de los países que hayan contribuido directa o indirectamente a la explotación de la Luna.

En el análisis de estos principios se puede decir que lo que se busca es una explotación sostenible de los recursos que se descubran en la Luna, es decir, que no agoten de manera indiscriminada y, como ya se venía mencionado, una consideración especial a aquellos países a los cuales se les dificulta el acceso al espacio y por ende a la Luna, para poder contar con las ventajas que provengan de estos descubrimientos (Demac, 1988).

Este régimen resultaría ser novedoso e interesante, ya que generaría la responsabilidad en los Estados que han accedido a la Luna de compartir su conocimiento y de lograr una exploración y explotación ordenada de estos recursos, algo que se oponía a un régimen de derecho de conquista que establecía que era posible explotar todo tipo de recursos que se extraigan de este territorio descubierto o conquistado. Es como si al imperio español se le hubiese obligado a realizar una explotación ordenada y sostenible del oro y la plata que descubrieran en América, al mismo tiempo que esta actividad beneficiara a los países más pobres, algo inconcebible en ese momento. Este es el impacto que tiene denominar a la Luna y otros cuerpos celestes como patrimonio de la humanidad y como objetos no apropiables. Este sería uno de los puntos que mayor discusión y debate generaría especialmente en los Estados Unidos, que en un principio impulsaba la propuesta, y la URSS, que se negaba rotundamente a la misma (Verschoor y Kopal, 2008, p. 51).

\section{EL ARTÍCULO $11^{\text {Ro }}$ DEL TRATADO DE LA LUNA: LOS RECURSOS NATURALES DE ESTE CUERPO COMO PATRIMONIO DE LA HUMANIDAD}

Si bien, desde el Tratado del 67 se consideraba que el espacio ultraterrestre, incluida la Luna y otros cuerpos celestes, eran res communis humanitatis, el término patrimonio común de la humanidad no había sido acuñado expresamente en ningún otro tratado del espacio. La utilización de este concepto en el Tratado de la Luna, para describir particularmente la naturaleza de la Luna y sus recursos naturales, fue entonces novedosa pero también controversial por la diversidad de significados e implicaciones que los distintos países le han otorgado. El término, de hecho, es una de las razones principales por las que Estados Unidos no ha ratificado el Tratado de la Luna y una de las razones por la que la Unión Soviética se opuso a su aprobación por muchos años. 


\section{A. El debate sobre el significado del tér- mino patrimonio común de la humani- dad como una manifestación de la ten- sión entre países desarrollados y en vía de desarrollo}

El concepto patrimonio común de la humanidad que se usó para describir la Luna y sus recursos naturales, fue propuesto por la delegación argentina ante la COPUOS en 1970 (Gangale, p. 70) y desde un principio fue el punto que más dificultó las negociaciones del Tratado de la Luna. Las negociaciones del Tratado de la Luna fueron el reflejo de las tensiones existentes en la época entre países en desarrollo (o nuevas económicas emergentes) y países desarrollados a propósito del control sobre recursos naturales en áreas o territorios que no podían ser objeto de apropiación por soberanía (tales como en alta mar o la Luna) ${ }^{7}$.

Por un lado, los países en desarrollo, entre ellos Brasil, Colombia y Venezuela, (que se habían agrupado en el grupo conocido como “G-77"), creían que el estatus que se le diera a los recursos naturales de la Luna y otros cuerpos celestes consistía en un paso importante para alcanzar un nuevo orden económico internacional o NIEO (New International Economic Order) (Gangale, 2009, p. 68). El G-77 quería poder participar en los beneficios que se derivaran de la explotación de los recursos extraterrestres, o por lo menos asegurarse de que podrían explotarlos en el futuro, cuando tuvieran la capacidad

7 Al mismo tiempo que se negociaba el Tratado de la Luna se estaba negociando el Tratado de Naciones Unidas sobre derecho del Mar o UNCLOS III (por sus siglas en inglés: United Nations Conventions on the Law of the Sea). técnica y económica para hacerlo, por lo que les interesaba que no se permitiera la propiedad privada sobre los recursos naturales de la Luna. Por el otro lado, potencias espaciales como Rusia y EE.UU. veían en la calificación de los recursos naturales de la Luna como patrimonio común de la humanidad, un impedimento para su futura explotación y para el establecimiento de un régimen comercial bajo el cual los recursos sí pudieran ser propiedad privada.

De hecho, si bien en un comienzo EE.UU aceptaba la cláusula de patrimonio común de la humanidad, gracias a lo cual el Tratado de la Luna pudo ser aprobado por consenso en la COPUOS, esta misma cláusula impidió que el tratado fuera posteriormente ratificado por los EE.UU. debido a la presión política y el lobby ejercido ante el Congreso por parte de sociedades espaciales que veían el tratado como un obstáculo a sus ambiciones de utilización de los recursos naturales de la Luna. Para éstas y otros opositores del Tratado de la Luna, el término patrimonio común de la humanidad implicaba entregar el control sobre los recursos del espacio ultraterrestre a los países del tercer mundo, ${ }^{8}$ así como también significaba que todos los Estados del mundo, incluyendo aquellos que no invirtieron

8 "Finally Mr. Chairman, let me say that, in the context of this treaty there is absolutely no justification for conceding to Third World control the resources of our solar system. If we sign the treaty and ratify it , we will be sacrificing an interest we cannon even calculate today in terms of the source of the world's resources in the next 100 years. Will they come form outer space, what will those resources be, and what happens to mankind's whole reach for outer space if we essentially put under an international socialist system the development of all resources in the solar system beyond the earth?" (United States House of Representatives, 1979, September 5 and 6, International space activities, 1979. Hearings before the Subcomitee on Space Science and Applications, Committee on Science and Technology. 96th Congress, 1st Session. Y4.sci2:96/50, p.116. Ctd. en Gangale, 2009, p 100). 
sus esfuerzos, mano de obra, capital o asumieron los riesgos para la explotación de dichos recursos, tendrían derechos sobre los mismos (Harlan, 2003).

De modo similar, la Unión Soviética, a pesar de su régimen socialista, se opuso a la cláusula de patrimonio común de la humanidad pues no quería que el tercer mundo tuviera alguna autoridad legal para dictaminar qué hacer con los recursos naturales extraterrestres extraídos a partir de la mano de obra soviética (Gangale, 2009, p. 71), al punto tal que:

se opuso a la inclusión de la frase patrimonio común de la humanidad hasta que se incluyó la segunda parte del párrafo 1 del artículo $11^{\text {ro }}$ en el que se indicaba que la Luna y sus recursos naturales era patrimonio común de la humanidad conforme lo dispuesto en este tratado, lo cual circunscribía drásticamente su significado (Gangale, 2009, p. 71).

Así las cosas, dados los distintos intereses en juego, para lograr la aprobación del Tratado de la Luna el legislador internacional prefirió no regular en ese momento las especificidades sobre un posible régimen para la explotación de los recursos naturales extraterrestres ni entrar a discutir el problema de si una vez explotados los recursos serían propios o no. En su lugar, prefirió que el texto reflejara una solución temporal. El Tratado de la Luna se aprobó incluyendo el término patrimonio común de la humanidad en el párrafo 1 del artículo $11^{\circ}$ pero circunscribiendo su significado al resto del Tratado y, particularmente, a la obligación de que en el futuro se estableciera un régimen internacional espe- cífico para regir la explotación de los recursos naturales de la Luna cuando dicha explotación estuviese a punto de llegar a ser viable (Tratado de la Luna, Art.11. 5).

Así pues, el texto final aprobado fue el siguiente: "La Luna y sus recursos naturales son patrimonio común de la humanidad conforme a lo enunciado en las disposiciones del Acuerdo y en particular en el párrafo 5 del artículo 11". El párrafo 5 del artículo $11^{\text {ro }}$ a su vez dispone que:

Los Estados Partes en el presente Acuerdo se comprometen a establecer un régimen internacional, incluidos los procedimientos apropiados, que rija la explotación de los recursos naturales de la Luna, cuando esa explotación esté a punto de llegar a ser viable. Esta disposición se aplicará de conformidad con el artículo 18 del presente Acuerdo. (Tratado de la Luna, 1979, Art. 11, párr. 11. Cursivas nuestras).

De esta manera, el Tratado de la Luna establece que mientras la explotación de los recursos naturales extraterrestres todavía no sea factible y no se establezca un nuevo régimen internacional, éstos deberán ser considerados patrimonio común de la humanidad, junto con la Luna y todas sus superficies, el resto del espacio ultraterrestre y demás cuerpos celestes. 


\section{B. El significado de la expresión patri-} monio común de la humanidad está dado por las disposiciones mismas del Tratado de la Luna, su objeto y fin, y está circunscrito al desarrollo de un nuevo régimen internacional que rija la explotación de recursos naturales

El significado de la expresión patrimonio común de la humanidad según las reglas de interpretación de los tratados enunciadas en la Convención de Viena sobre Interpretación de los tratados (artículo 31 ${ }^{\text {ro }}$ ) debe estar dada en primer lugar por una interpretación de buena fe y conforme al sentido corriente que haya de atribuirse a los términos del tratado en el contexto mismo de éste y teniendo en cuenta su objeto y fin. Siguiendo lo anterior, el término patrimonio común de la humanidad en su acepción común implica que la Luna y sus recursos pertenecen a todas las personas y, por tanto, no son apropiables por ningún país ni individuo en particular. El término patrimonio común de la humanidad está entonces relacionado con el principio de no apropiación, pues implica precisamente la imposibilidad de establecer derechos de propiedad sobre la Luna y sus recursos naturales.

En concordancia con lo anterior, Edward R. Finch y Amanda Lee Moore (Ctd. en Gangale, 2009) delegados ante en la COPUOS, señalan que:

la esencia de la expresión patrimonio común de la humanidad en el contexto del Tratado de la Luna recae en la existencia de un patrimonio común, esto es, según ellos, en la igualdad de derechos para explorar y usar la Luna y sus recursos naturales. No implica, sin em- bargo, criterios específicos de implementación o procedimientos. Para la profesora Eiline M. Galloway del Instituto Internacional de Derecho del Espacio (IISL), el término patrimonio común de la humanidad, con respecto a la Luna y sus recursos naturales, implica que toda persona en el mundo tiene un interés respecto a este patrimonio." (p. 74).

Sin embargo, también señala que este concepto está limitado en su implementación en la medida que el artículo $11^{\text {ro }}$ del tratado señala claramente que "la Luna y sus recursos naturales son patrimonio común de la humanidad conforme a lo enunciado en las disposiciones del presente Acuerdo y en particular en el párrafo 5 del presente artículo" (Galloway Ctd. En Gangale, 2009, p. 74).

En efecto, como señala Galloway (Ctd. en Gangale 2009 ) el artículo $11^{\text {ro }}$ párrafo 1 señala que: “la Luna y sus recursos naturales son patrimonio común de la humanidad conforme a lo enunciado en las disposiciones del presente Acuerdo y en particular en el párrafo 5 del presente artículo" (Asamblea General de las| Naciones Unidas, 1979, Acuerdo que debe regir las actividades de los Estados en la Luna y otros cuerpos celestes. Cursivas y subrayado nuestro), lo cual quiere decir que la expresión patrimonio común de la humanidad no puede ser interpretada de manera independiente o aislada del resto del Tratado de la Luna y sin tener en cuenta la obligación por parte de los Estados Parte de establecer un régimen internacional que rija particularmente la explotación de los recursos naturales de la Luna cuando ésta esté a punto de ser viable. 
Esto también significa que cualquiera sea el significado o interpretación dada a esta misma expresión en un contexto distinto (tal como en el Tratado de Naciones Unidas sobre derecho del Mar o UNCLOS III) resulta irrelevante para el contexto espacial. Por esto mismo, no es cierto que el término patrimonio común de la humanidad en el Tratado de la Luna tenga el mismo significado al atribuido en el Tratado UNCLOS III, como se empeñan en señalar algunas personas. No solo el UNCLOS III y el Tratado de la Luna regulan medios totalmente distintos (alta mar y la Luna, respectivamente), sino que son tratados con diferentes propósitos, ámbitos de aplicación y estructura (Gangale, 2009, p. 102). EI UNCLOS III, al señalar que los recursos naturales en el fondo del mar en alta mar son patrimonio común de la humanidad, les atribuye un régimen bastante detallado según los fines y propósitos de ese tratado en particular (Gangale. 2009, p. 102). Por ejemplo, el UNCLOS III, en sus artículos 156 - 158 dispone que debe haber una autoridad que gobierne o guíe la extracción de los recursos del fondo del mar (Autoridad Internacional de los Fondos Marinos ${ }^{9}$ ), lo cual, según lo dicho anteriormente, no es igualmente aplicable para la explotación de los recursos naturales de la Luna.

Teniendo en cuenta todo lo anterior, la expresión patrimonio común de la humanidad, en el Tratado de la Luna, significa que la Luna y sus re-

$9 \quad$ La Autoridad Internacional de los Fondos Marinos es la organización por conducto de la cual los Estados Partes organizarán y controlarán las actividades en la Zona de conformidad con esta Parte, particularmente con miras a la administración de los recursos de la Zona. (Tratado de Naciones Unidas sobre el Derecho del Mar , 1982, Art.157, num. $1^{\circ}$. cursos naturales pertenecen a todos los países, lo cual implica que todos ellos tienen igualdad de derechos para su exploración y utilización, basándose en la cooperación internacional y reciprocidad. Esta concepción, no obstante, está circunscrita a la posibilidad de que en el futuro se cree un régimen internacional para la explotación de dichos recursos que permita obtener derechos de propiedad sobre los mismos.

Finalmente se quiere aclarar que los materiales que se han traído de la Luna y otros cuerpos celestes de regreso a la Tierra hasta el momento, son apenas muestras de los minerales y otras sustancias lunares o extraterrestres que realmente no representan una explotación con fines comerciales de recursos naturales ni cantidades significantes. Sobre estas muestras, según señala el artículo $6^{\text {to }}$ del Tratado de la Luna, sí pueden recaen derechos de propiedad y podrán ser utilizadas con fines científicos. En efecto, el mencionado artículo $6^{\text {to }}$ señala que:

Al realizar investigaciones científicas con arreglo a las disposiciones del presente Acuerdo, los Estados Partes tendrán derecho a recoger y extraer de la Luna muestras de sus minerales y otras sustancias. Esas muestras permanecerán a disposición de los Estados Partes que las hayan hecho recoger y éstos podrán utilizarlas con fines científicos.

Dicha disposición también señala la conveniencia de poner parte de esas muestras a disposición de otros Estados Parte para la investigación científica, pero no establece ninguna obligación de compartir dichas muestras. 


\section{PROBLEMAS ESPECÍFICOS RELATIVOS AL TRATADO DE LA LUNA}

\section{A. ¿Cuál es la fuerza vinculante del Tratado de la Luna?}

Teniendo en cuenta que ninguno de los Estados que actualmente desarrollan gran parte de la la actividad espacial -si no toda-, o que han llegado a Luna y a otros cuerpos celestes, han ratificado el Tratado de la Luna, vale la pena preguntarse cuál es su verdadera utilidad práctica y qué representa para el Derecho Espacial.

El Acuerdo que debe regir las actividades de los Estados en la Luna y otros cuerpos celestes es un Tratado internacional multilateral jurídicamente vinculante vigente desde 1984, año en que fue depositado el quinto instrumento de ratificación. Sin embargo, ha sido ratificado por una pequeña minoría de países (la mayoría de los cuales no tiene capacidad para ir al espacio por su cuenta), y ni los EE.UU, ni Rusia, o China, son actualmente Parte del mismo, lo cual, no solo le resta fuerza política al mismo, sino que inevitablemente, conduce a cuestionar su utilidad para el derecho espacial. No siendo Parte estos países, en principio, no estarían obligados a seguir sus disposiciones en el desarrollo de sus actividades espaciales en la Luna y otros cuerpos celestes.

No obstante, el hecho que el Tratado de la Luna desarrolle principios ya enunciados en el Tratado del 67, y haya habido, lo que consideramos ha sido una aceptación tácita del Tratado de la
Luna por parte de la comunidad internacional en los términos descritos a continuación, le otorga al Tratado una fuerza vinculante más grande de la esperada.

Para empezar, el hecho de que exista una regulación específica para regir las actividades de los Estados en la exploración de la Luna y otros cuerpos celestes, con el carácter de tratado internacional y no de mera declaración o resolución no vinculante es ya un gran avance, pues significa que la comunidad internacional consideró que era un tema que necesitaba ser regulado y convertido en "hard law". Es más, el hecho de que el Tratado de la Luna hubiese sido aprobado por consenso en el ámbito del Comité para el Uso Pacífico del Espacio ultraterrestre (en adelante COPUOS por sus siglas en inglés) y luego adoptado por votación mediante resolución de la Asamblea General de las Naciones Unidas es un gran indicador de la voluntad de los Estados en adoptar tal regulación y de que quedarán establecidas una reglas vinculantes al respecto, así después decidieran por motivos políticos, prácticos o simple desinterés, no ratificar el Tratado.

Además, dado que las disposiciones del Tratado de la Luna en su mayoría se limitan a desarrollar principios ya recogidos en el Tratado el Espacio Ultraterrestre, incluso si el Tratado de la Luna no ha sido ratificado sino por quince países ${ }^{10}$, se entiende que sus provisiones son vinculantes

10 Según la Oficina de asuntos legales de las Naciones Unidas son Partes del Tratado de la Luna: Australia, Austria, Bélgica, Chile, Kazakhstán, Líbano, México, Marruecos, los Países Bajos, Pakistán, Perú, Filipinas, Arabia saudita, Turquía y Uruguay. (United Nations Treaty Collection, 2013). 
para la comunidad internacional que sí han ratificado el Tratado del Espacio Ultraterrestre, que son, para julio de 2013, ciento un Estados ${ }^{11}$.

Sumado a lo anterior, algunas de las disposiciones del Tratado de la Luna pueden incluso llegar a ser consideras como costumbre internacional derivada de un tratado, en los términos del artículo 38vo de la Convención de Viena sobre derecho de los tratados, ${ }^{12}$ en la medida que, gran parte de su articulado refleja los mismos principios ya reseñados en el Tratado del Espacio Ultraterrestre, los cuales gozan de una amplia aceptación por la comunidad internacional y algunos incluso son efectivamente reconocidos como costumbre internacional. Ejemplo de las disposiciones en el Tratado de la Luna que son consideradas como Derecho Internacional Consuetudinario son el artículo 2 do, que señala la necesidad de la conformidad con el Derecho Internacional y la Carta de las Naciones Unidas en las actividades que se desarrollen en la Luna, el artículo 3ro que indica el principio de uso pacífico y no utilización de armas nucleares en la Luna o en sus órbitas, el artículo 4to que señala que la exploración y utilización de la Luna incumbe a toda la humanidad y se efectuará en provecho e interés de todos los países, el artículo 12 do sobre la propiedad de los objetos espaciales y la responsabilidad internacional de los

11 De conformidad con la información disponible en la página web de la Oficina de las Naciones Unidas para asuntos del espacio exterior a la fecha. (UNOOSA, Treaty Database: Treaty Signatures).

12 Artículo 38vo: "Normas de un tratado que lleguen a ser obligatorias para terceros Estados en virtud de una costumbre internacional. Lo dispuesto en los artículos 34 a 37 no impedirá que una norma enunciada en un tratado llegue a ser obligatoria para un tercer Estado como norma consuetudinaria de derecho internacional reconocida como tal"
Estados por los daños causados por sus objetos espaciales y por las actividades nacionales que realicen en el espacio ultraterrestre, la Luna y otros cuerpos celestes las entidades no gubernamentales y el artículo 11.2 del Tratado de la Luna que consagra la obligación de no apropiación nacional mediante reclamaciones de soberanía, por medio del uso o la ocupación, ni por ningún otro medio.

Prueba de lo anterior es que los Estados que efectivamente han llegado a la Luna y otros cuerpos celestes no han intentado reclamar soberanía sobre los mismos y que, incluso durante la Guerra fría se evitó el uso bélico del espacio. Es más, si bien ningún Estado de lanzamiento ha ratificado el Tratado de la Luna, éstos tampoco han objetado su existencia en el plano internacional, no se han opuesto a que otros países lo firmen, ni han ejercido una oposición persistente para evitar que sus disposiciones se conviertan en costumbre internacional, lo cual supone que ha habido una aceptación tácita del tratado.

No obstante, es de notar que el Tratado de la Luna, probablemente gracias a la existencia de otros tratados del espacio que recogen disposiciones similares, ha permanecido hasta el momento prácticamente inutilizado o en un estado de letargo. Incluso el artículo 11ro del instrumento, que se refiere al régimen de explotación de los recursos naturales en la Luna y otros cuerpos celestes, quizá la única disposición novedosa en el mismo, ha permanecido sin mayor mención en el plano internacional gracias a que se ha entendido como un régimen temporal 
para los recursos naturales extraterrestres que rige mientras las actividades de explotación de dichos recursos no sean factibles.

Así las cosas, el verdadero desafío para el Tratado de la Luna solo llegará en el momento en que la explotación de recursos naturales en la Luna y otros cuerpos celestes esté a punto de ser viable y la actividad espacial y exploración de la Luna tenga propósitos más allá de los meramente científicos. En efecto, cuando los intereses comerciales y económicos de los Estados y de las compañías privadas estén en juego y ante la voracidad de los mismos, será el momento en que se podrá medir la verdadera utilidad práctica del Tratado de la Luna y se verá si sus disposiciones, particularmente el artículo 11ro, serán respetadas o reemplazadas por ambiciones particulares (por ejemplo, estableciendo la propiedad sobre los terrenos de donde se obtendrán los recursos naturales). De ahí la importancia de que, antes que la explotación de los recursos naturales esté a punto de ser factible, se desarrolle un régimen internacional relativo a la explotación de los recursos naturales en la Luna y otros cuerpos celestes que regule los procedimientos y detalles de dicha explotación.

\section{B. El Acuerdo que debe regir las activi- dades de los Estados en la Luna y otros cuerpos celestes: ¿Un inminente fraca- so? Análisis de las razones por las cuales la mayoría de Estados no han ratificado el Tratado de la Luna.}

Como ya se señaló el Tratado de la Luna solo ha sido ratificado por quince Estados y firmado por otros 4, pero: ¿cuál es la razón de esto? A pesar de que el Tratado de la Luna fue aprobado por consenso ( $y$ el consenso solo se obtiene cuando no hay ninguna objeción )en el marco de la Comisión de Utilización del Espacio UItraterrestre con Fines Pacíficos y aprobado por votación en la Asamblea General de las Naciones Unidas en diciembre de 1979, muchos de los países que se esperaba que lo ratificaran, especialmente Estados Unidos Y la Unión Soviética (que eran las dos potencias espaciales en la época y los únicos dos Estados que en el momento tenían posibilidades reales y técnicas de llegar a la luna y otros cuerpos celestes), no lo hicieron.

En primer lugar, podría señalarse como la razón principal por la que el Tratado de la Luna no ha sido ratificado, el hecho de que un análisis del articulado, muestra que, aparte del artículo 11ro, este instrumento no aporta material nuevo al ya contenido en el Tratado del $67 \mathrm{y} / \mathrm{o}$ del que ya está enunciado en anteriores tratados del espacio. Por tanto, es viable afirmar que su no ratificación no cambia sustancialmente el panorama del Derecho Espacial. No obstante, las razones por las cuales no se ha ratificado son muchas más.

Existe una razón práctica y es la disminución de las misiones exploratorias, tripuladas y no tripuladas, hacia la Luna y hacia otros cuerpos celestes luego de 1979 y hasta la actualidad, cuando por fin está siendo retomada. Para 1979, señala Thomas Gangale, las expediciones tripuladas a la Luna eran prácticamente nulas, de hecho, la última expedición lunar por parte de los Estados 
Unidos había sido siete años antes, en 1972 con el Apollo 17. De igual forma, para comienzos de los años ochenta los EE.UU. habían abandonado los programas diseñados para lograr un aterrizaje tripulado en Marte y la posibilidad de un programa de exploración lunar llevado a cabo completamente por humanos había sido totalmente descartado (Gangale, 2009, p. 84). Por su parte, la Unión Soviética había abandonado por completo su programa de aterrizaje lunar tripulado con humanos -mejor conocido como L3(Gangale, 2009, p. 84). Así pues, excepto por la puesta en órbita de algunas pocas sondas espaciales a su alrededor, no mucho ha pasado en la Luna desde 1979 por lo que no ha habido una urgencia de ratificar el Tratado de la Luna (Gangale, 2009, p.93).

Por otro lado, muchos Estados pensaron que era prematuro establecer un régimen jurídico que gobernara la extracción y explotación de los recursos naturales extraterrestres cuando dicha explotación todavía era una posibilidad muy limitada y que no se materializaría efectivamente sino hasta dentro de muchos años (Danilenko 1989, Ctd. en Gangale 2009, p. 85). Si bien es cierto que anticiparse a establecer una regulación pues ser muy útil para establecer un marco guía para futuras actividades espaciales, también es cierto que es peligroso depender mucho de dicha regulación, especialmente cuando es probable que ésta implique la necesidad de establecer reglas detalladas sobre temas técnicos y económicos y anticiparse al estado de la ciencia y la tecnología en el futuro (Danilenko, 1989, Ctd. en Gangale, 2009, p. 87).
La otra gran razón para no ratificar el Tratado de la Luna es el simple desinterés y falta de necesidad. Muchos Estados, especialmente los países en vía de desarrollo, no han visto la necesidad de ratificarlo porque no desarrollan actividades espaciales ni en la Luna y ni en otros cuerpos celestes. A esto se une que no han existido en la actualidad conflictos alrededor de la aplicación del tratado, por lo cual no existe una verdadera motivación para ratificarlo. El día que se presente un conflicto al respecto es probable que el interés de los países en ratificar el tratado aumente.

Finalmente, el motivo más relevante por el que no ha sido posible que muchos Estados de Ianzamiento ratifiquen el Tratado es que se han presentado en su contra objeciones a nivel nacional ${ }^{13}$. Esto es especialmente cierto para Ios EE.UU., en donde varias asociaciones del espacio, como la sociedad $\mathrm{L}^{14}{ }^{14}$, ejercieron un gran lobby político para evitar la aprobación del Tratado de la Luna en el Congreso, pues veían que su ratificación podía llegar a obstaculizar su agenda espacial a futuro. El objetivo de la sociedad espacial L5 era construir una colonia auto sostenible en un punto en el espacio conocido como Lagrange $L 5^{15}$, usando únicamente materiales lunares. La sociedad consideró que si los

13 En países que no desarrollan actividad espacial ni siquiera se ha planteado el debate a nivel interno de si conviene ratificar el Tratado de la Luna o no. En estos países la razón por la que no se ratifica se limita a que nunca ha estado en la agenda nacional por desinterés o falta de necesidad.

14 La Sociedad de L5 es un precursor de la Sociedad Espacial Nacional y promovió la posibilidad de establecer una colonia en los puntos alrededor del $L_{4} \circ L_{5}$ del sistema de Tierra. (Brandt-Erichsen, 1994).

15 "The Lagrange Points are positions where the gravitational pull of two large masses precisely equals the centripetal force required for a small object to move with them" (The Langrange Points, 2012). 
recursos naturales de la Luna fueran considerados por el Tratado de la Luna como patrimonio común de la humanidad, ello impediría el uso de materiales lunares para el establecimiento de su colonia L5 (Gangale, 2009, p. 75). Pronto otras organizaciones se unieron a la opiniones de la sociedad L5, tales como la Sociedad Futura Espacial, argumentando que el Tratado de la Luna era un obstáculo al futuro desarrollo económico de los EE.UU. con lo cual finalmente lograron impedir la aprobación del tratado en el Congreso de los EE.UU.

A pesar de todo lo anterior, en el 2012 dos Estados se adhirieron al Tratado de la Luna: Arabia Saudita y Turquía. Si bien es cierto que la ratificación del Tratado de la Luna por parte de Turquía o Arabia Saudita puede deberse al Ilamado de la COPUOS a que los Estados del mundo ratifiquen los tratados del espacio ${ }^{16}$, también puede que Turquía, en la medida que avanza en su actividad espacial, este interesada en contar con una regulación al respecto, especialmente en caso de que se presente algún problema en su ejercicio y que Arabia Saudita tenga una agenda espacial que hasta ahora se desconozca. De hecho, la Asociación Aeronáutica Turca (o THK por sus siglas en inglés), pretende lanzar el primer astronauta al espacio en el año 2023 para la celebración del centenario de la República de Turquía (Listner, 2011).

16 La COPUOS y las Naciones Unidas han exhortado a los países del mundo a que ratifiquen los tratados del espacio. En general la ONU exhortó a todos los países del mundo a ratificar todos los tratados internacionales multilaterales.
Esto muestra que a medida que los países en desarrollo avancen en sus políticas internas sobre el espacio ultraterrestre o implementen políticas espaciales propias, les será más importante tener un instrumento vinculante que regule el tema de la actividad espacial en la Luna y otros cuerpos celestes al que puedan acudir en caso de presentarse problemas. De igual forma, a medida que crezca la economía y se desarrollen programas espaciales nacionales en los países en vía de desarrollo, la idea de ser Parte de un tratado del espacio será más llamativa y con el tiempo considerarán que es necesario contar con estos instrumentos jurídicos. Así pues, si bien las mayores potencias espaciales en actualidad como China, Rusia y EE.UU. no han firmado el Tratado de la Luna, el que poco a poco más países lo ratifiquen (así estén apenas entrando a la era especial), le dará un mayor peso y fuerza política al Tratado.

Si bien el interés de los EE.UU. de volver a enviar misiones a la Luna (en el 2004 el Presidente de Ios Estados Unidas George W. Bush había anunciado el retorno a la Luna ${ }^{17}$ ) se ha visto frustrado por la reciente crisis económica y los ajustes fiscales que ha emprendido esta nación (RTVE, 2010), aún persisten intereses similares por parte de Rusia (Zak, 2008), China, Japón, India y Corea del Sur (Lyall \& Larsen, 2009, p. 175). ${ }^{18}$

17 De hecho, dentro de la agenda del programa espacial "constelación" de la NASA, Estados Unidos pretendía volver a tener astronautas en la Luna para el 2020 (The vision for space exploration, 2004).

18 El 14 de septiembre de 2007, los japoneses lanzaron al espacio su sonda espacial, Kaguya (o también llamada Selena), la cual se estableció en órbita lunar con el fin de recoger información sobre la topografía, geología y ambiente lunar. Por otro lado, si bien se desconocen los planes de China hacia el futuro, desde noviembre de 2007, China tiene una sonda espacial orbitando la Luna. Corea del Sur, por su par- 
Esto sugiere que se aproxima el momento en el que el Tratado de la Luna cobrará relevancia en el escenario mundial.

Bajo estos términos se puede afirmar que el Tratado de la Luna no está muerto ni ha fracasado; simplemente se encuentra en un estado de letargo del cual puede despertar a medida que más países incursionen en la actividad espacial y otros retomen su interés por las misiones lunares y a otros cuerpos celestes. Por esto mismo es tan importante que desde ya exista un régimen jurídico internacional que rija las actividades de los Estados en la Luna y otros cuerpos celestes al que se pueda acudir a medida que esto suceda.

\section{C. ¿El Acuerdo también prohíbe la pro- piedad privada sobre la Luna u otros cuerpos celestes?}

\section{Casos de reivindicaciones de soberanía por parte de particulares y compañías privadas}

A pesar de que el artículo $2^{\text {do }}$ del Tratado del $67^{19}$ y el párrafo $2^{\text {do }}$ del artículo $11^{20}$ del Acuer-

te, tiene planes para enviar un vehículo espacial a la Luna para el año 2020 y planea un aterrizaje para el 2015. Por último, India lanzó su misión Lunar Chadrayan-1 el 23 de octubre de 2008. Ésta orbitará la Luna y eventualmente enviará una sonda espacial a la superficie para preparar el aterrizaje en la misma. (Lyall \& Larsen, 2009, p.175-199).

19 "El espacio ultraterrestre, incluso la Luna y otros cuerpos celestes, no podrán ser objeto de apropiación nacional por reivindicación de soberanía, uso u ocupación, ni de ninguna otra manera" (Tratado de la Luna, 1967, Art. 2).

20 "La Luna no puede ser objeto de apropiación nacional mediante reclamaciones de soberanía, por medio del uso o la ocupación, ni por ningún otro medio" (Tratado de la Luna, 1967, Art. 11.2). do sobre la Luna consagran el principio de no apropiación sobre la Luna y otros cuerpos celestes, esto no ha impedido que particulares y compañías privadas reivindiquen supuestos derechos de propiedad sobre la Luna.

Uno de los casos más famosos es el del estadounidense Denis Hope quien en 1980: envió cartas al gobierno de los Estados Unidos, a la Embajada de la Unión de Repúblicas Socialistas Soviéticas (URSS )en Washington y a la sede de las Naciones Unidas, declarando tener propiedad sobre la Luna y todos los planetas del sistema solar. (Mejía, 2008, p.174).

Y prontamente argumentó haber registrado su propiedad sobre la Luna en el Estado de California de los EE.UU., Hope también creó la compañía privada Embajada Lunar, con presencia no solo en EE.UU. sino también en Australia, Japón, Nueva Zelanda, Reino Unido e Irlanda a través de la cual se hizo millonario por la venta de terrenos de la Luna. "En la página electrónica de la Embajada Lunar se indica que todas estas transacciones son legales y están de acuerdo con el Tratado del Espacio" (Mejía, 2008, p. 174). Denis Hope, además aseguraba públicamente que "el Tratado del Espacio no se le aplica a él como persona física, sólo a los gobiernos, por lo que no estaba obligado a respetarlo" (Mejía, 2008, p.179). Si bien las autoridades de EE.UU. no reconocen ni respaldan tales declaraciones de apropiación y no hay evidencia de que Hope u otras compañías efectivamente tengan registro de la propiedad sobre la Luna, sus declaraciones no han sido llevadas ante ninguna corte federal en los EE.UU. (Mejía, 2008, p. 188). No 
obstante, en otros países como China, el caso de la compañía Embajada lunar sí ha sido objeto de fallo judicial. En efecto, en 2007 la Corte de Primera Instancia de Beijing señaló que:

"la República Popular China había accedido al Tratado del Espacio desde 1983, el cual prohibía la apropiación de la Luna, por lo que ningún individuo o Estado podía tener propiedad sobre este cuerpo celeste"

y condenó a la compañía embajada lunar por fraude, ordenó que se regresara el dinero a los clientes que habían pagado y además la multó (Mejía, 2008, p.185. Cursivas en el original).

\section{La imposibilidad de establecer propiedad privada en la luna y otros cuerpos celestes}

Dicho todo lo anterior se debe explicar por qué las reivindicaciones de propiedad sobre la Luna y otros cuerpos celestes no son viables y carecen de fundamento alguno desde la perspectiva del Derecho Espacial.

Como ya se señaló, el Tratado del 67 en su artículo II indica que "El espacio ultraterrestre, incluso la Luna y otros cuerpos celestes, no podrán ser objeto de apropiación nacional por reivindicación de soberanía, uso u ocupación, ni de ninguna otra manera", lo cual es reiterado por Tratado de la Luna en su artículo 11.2. y 11.321. La obligación de no apropiación es en-

21 : "Ni la superficie ni la sub-superficie de la Luna, ni ninguna de sus partes o recursos naturales podrán ser propiedad de ningún Estado, organización internacional intergubernamental o no gubernamental, organización nacional o entidad no gubernamental ni de ninguna persona física" (Art 11, párr. 3). tonces "Hard Law" y vinculante para los Estados que han ratificado estos tratados. Incluso aquellos Estados que no los han ratificado, deben cumplir con la obligación de no apropiación en virtud de que, los principios del Tratado del 67, incluyendo el principio de no apropiación, han pasado a ser concebidos como costumbre internacional. Así pues, cualquier aceptación o reivindicación de soberanía sobre el espacio ultraterrestre, incluida la Luna y otros cuerpos celestes, implica no solo una violación al Tratado del 67 y al Tratado de la Luna, sino a la costumbre internacional.

No obstante, esto no resuelve el argumento utilizado por Dennis Hope para asegurar la legitimidad de su supuesto derecho de propiedad en la Luna, según el cual el principio de no apropiación, al ser una obligación consagrada en un tratado internacional, es solo predicable respecto a los Estados pero no frente a los particulares. A continuación se expondrán entonces los motivos por los cuales tal argumento es inviable:

1.) El Artículo 31.1 de la Convención de Viena sobre el derecho de los tratados señala que un tratado deberá interpretarse de buena fe conforme al sentido corriente que haya de atribuirse a los términos del tratado en el contexto de estos y teniendo en cuenta su objeto y fin. Por esto hay que tener en cuenta, a )el texto mismo de del artículo $11^{\text {ro }} \mathrm{y}, \mathrm{b}$ )se debe examinar cuál era el propósito y fin que se tuvo al incluir el principio de no apropiación en la Resolución 1962 de 1963, en el Tratado del Espacio Ultraterrestre y luego en el Tratado de la Luna. 
Quienes argumentan que el principio de no apropiación no es aplicable respecto de compañías privadas o particulares ignoran que según el texto mismo del Tratado, la Luna y otros cuerpos celestes no podrán ser objeto apropiación por reivindicación de soberanía, uso u ocupación, ni de ninguna otra manera", lo cual conforme al sentido corriente, implica cualquier forma de apropiación, incluyendo obviamente reivindicaciones de soberanía por organizaciones no gubernamentales o particulares. El propósito de incluir el principio de no apropiación en un cuerpo normativo internacional era principalmente evitar que se siguiera aplicando el derecho de conquista que habían imperado en las actividades de descubrimiento llevadas a cabo en la Tierra para las cosas consideradas res nullius, así como evitar que el espacio ultraterrestre, incluida la Luna y otros cuerpos celestes, se convirtieran en otro escenario más de la Guerra Fría. Siendo esto así, no hay duda que lo que quiso el legislador internacional era evitar cualquier forma de apropiación en el espacio, la Luna y otros cuerpos celestes incluyendo cualquier forma de apropiación por parte de particulares. Esto puede ser constatado al revisar los antecedentes inmediatos del Tratado del Espacio Ultraterrestre que son las resoluciones 1721 de $1960^{22}$ y

22 "Ante la posible amenaza que presentaba el uso del espacio para la guerra y auge actividades espaciales: En la asamblea General de las Naciones Unidas en 1961 se aprobó por consenso una resolución (Resolución 1721(XVI), 20 Diciembre de 1961 )en la cual se recomendó que, en sus actividades espaciales, los Estados se guiaran por dos principios fundamentales, a saber, que el derecho internacional, incluida la Carta de Naciones Unidas, se aplica al espacio ultraterrestre y a los cuerpos celestes y que el espacio ultraterrestre y los cuerpos celestes podrán ser libremente explorados y utilizados por todos los Estados de conformidad con el derecho internacional y no podrá ser objeto de apropiación" (Kopal, 2009, p.2).
1962 de 1963 donde ya se mencionaba el principio de no apropiación.

2.) Es lógico que cuando un Estado se obliga por medio de un tratado, deba asegurarse que su población también lo cumpla, de lo contrario un tratado internacional nunca sería más que una imposición teórica a un sujeto inexistente (el Estado) cuyas obligaciones nunca podrían verse materializadas.

3.) El artículo $6^{\text {to }}$ del Tratado del 67 y el artículo $14^{\text {to }}$ del Tratado de la Luna, señalan que los Estados son responsables internacionalmente de las actividades que sus organizaciones no gubernamentales realicen en la espacio ultraterrestre, incluida la Luna y otros cuerpos celestes, por lo que deben asegurarse que las actividades espaciales nacionales, incluidas las de las organizaciones no gubernamentales, sean llevadas a cabo de conformidad con las obligaciones y disposiciones del respectivo Tratado. Señalan además que todas las actividades de las entidades no gubernamentales en el espacio ultraterrestre, incluso la Luna y otros cuerpos celestes, deberán ser autorizadas y fiscalizadas constantemente por el pertinente Estado Parte en el Tratado. Esto significa que cualquier Estado que reconozca o acepte reivindicaciones de propiedad sobre la Luna por parte de sus nacionales estaría incurriendo en una violación del artículo $6^{\text {to }}$ del Tratado del $67 \mathrm{y}$ del artículo $14^{\text {to }}$ del Tratado de la Luna, por cuanto establecen que el Estado debe no solo autorizar, vigilar y fiscalizar las actividades de entidades no gubernamentales en el espacio ultraterrestre, la Luna y otros cuerpos celestes, sino que también debe 
asegurarse que éstas se lleven a cabo de conformidad con las obligaciones del tratado ${ }^{23}$.

4.) Teniendo en cuenta que: i) la actividad espacial debe emprenderse con la autorización y a través de un Estado de lanzamiento, el cual a su vez tiene la obligación de asegurarse y de cumplir las disposiciones del Tratado del Espacio Ultraterrestre y la costumbre internacional; y que además es responsable por las actividades nacionales que realicen los organismos no gubernamentales en el espacio, la Luna y otros cuerpos celestes y, ii) que toda actividad espacial implica también un Estado de registro, ante el cual se iniciará una reclamación por eventuales daños causados por un objeto espacial, se puede afirmar que cualquier intento de apropiarse físicamente de la Luna u otro cuerpo celeste por parte de un particular u organización no gubernamental sería inviable sin la ayuda, o por lo menos la aceptación de un Estado, quien al colaborar para tales fines estaría violando las disposiciones de los tratados internacionales y la costumbre internacional en el Derecho del Espacio.

En conclusión el espacio ultraterrestre, la Luna y otros cuerpos celestes no pueden ser objeto de ningún tipo de apropiación, prohibición que aplica tanto a Estados como a particulares, compañías privadas o cualquier otro tipo de entidad o asociación entre estos.

23

"Some have argued that Outer Space treaty's broad definitions allow individual appropiation of space and celestial bodies because it only specifically prohibits appropriation by States, however, states are responsible for the actions of individuals, and property claims must occur through the State's laws. Therefore individual may not claim space or celestial bodies" (Gangale, 2009 p. 37).

\section{LA REGULACIÓN SOBRE LA LUNA Y OTROS CUERPOS CELESTES EN EL FU- TURO: ¿QUÉ SE ESPERA?}

Consideramos que el Tratado de la Luna debe ser desarrollado con una mayor profundidad, no sólo para incluir algunas definiciones que le serían útiles, tales como qué se entiende por cuerpo celeste, sino para actualizar y adaptar las disposiciones del Tratado a las nuevas tecnologías, avances de la ciencia y dinámicas de exploración y utilización de la Luna y sus recursos naturales.

Actividades tales como el turismo espacial ya son una realidad. En 2001, el estadounidense Dennis Tito se convirtió en el primer turista espacial al pagar 20 millones de dólares para ir a la Estación Internacional Espacial, donde permaneció ocho días, luego de los cuales regresó a la Tierra a bordo del vehículo espacial ruso SOYUZ (Wall, 2012). Desde entonces, siete personas más han ido como turistas al espacio. Con el recién desarrollado interés de algunas potencias espaciales de impulsar exploraciones lunares, (léase China), e incluso de enviar misiones tripuladas, no será dentro de mucho que el turismo espacial a la Luna podrá ser una realidad. Este tipo de actividades deberán entonces ser tenidas en cuenta por el Tratado de la Luna, pues si bien ya existe un tratado internacional relativo a los astronautas de 1967, la particularidad de una actividad como el turismo espacial, cuyos operadores tendrán objetivos meramente comerciales, amerita que se regule o adapte el Tratado de la Luna para incluir esta nueva actividad. 


\section{A. Propuestas para un futuro régimen jurídico internacional que rija la explota- ción de los recursos naturales extraterrestres}

El Tratado de la Luna en el artículo 11.5 señala la obligación de las Partes de establecer un régimen internacional que rija la explotación de los recursos naturales en la Luna cuando está explotación esté a punto de ser viable. Si bien dicha explotación todavía no es una realidad, el tema cobra cada vez más relevancia en la medida que se ha confirmado la presencia de agua congelada (Farrel \& Neil-Jones, 2012) y de sustancias como hidrógeno comprimido en la Luna (Teitel, 2012). Estos hallazgos ponen de presente la necesidad de establecer este nuevo régimen internacional que se adelante a regular el tema. A pesar de que el Tratado no establece ningún principio o norma específicos para este nuevo régimen internacional sí señala en el párrafo 7 del artículo $11^{\text {ro }}$, que se debe incluir entre sus principales finalidades, por lo menos: a) el desarrollo ordenado y seguro de los recursos naturales de la Luna; b) la ordenación racional de esos recursos; c) la ampliación de las oportunidades para el uso de esos recursos; $d$ ) una participación equitativa de todos los Estados Parte en los beneficios obtenidos de esos recursos, teniéndose especialmente en cuenta los intereses y necesidades de los países en desarrollo, así como los esfuerzos de los países que hayan contribuido directa o indirectamente a la explotación de la Luna.

Teniendo en cuenta lo anterior, a continuación se plantearán las bases para un posible régi- men internacional o Tratado Internacional que rija esta eventual explotación de recursos naturales en la Luna:

1.) En primer lugar, que este nuevo régimen debe estar en armonía con las disposiciones del Tratado de la Luna y de los demás tratados del espacio precedentes, por lo cual consideramos fundamental que tenga como base el principio de cooperación y el principio de uso pacífico del espacio. Así como el artículo $9^{\text {no }}$ del Tratado del 67 dispone que los Estados en "todas sus actividades en el espacio ultraterrestre, incluso la Luna y otros cuerpos celestes deberán tener debidamente en cuenta los intereses de los demás Estados Partes en el tratado" (Cursivas nuestras). Consideramos que los Estados que emprendan una actividad de explotación de los recursos naturales en la Luna deben tener en mente el interés de los demás Estados en dicha actividad. Esto implica que debe haber una cooperación y ayuda permanente entre los diversos Estados y organizaciones que pretendan explotar los recursos extraterrestres. Dicha cooperación debe tener como base la reciprocidad. En armonía con lo anterior, consideramos que el nuevo régimen jurídico debe contemplar que esos recursos se usen exclusivamente en beneficio de la humanidad. Esto quiere decir que estos nuevos recursos naturales no podrían ser usados para construir armas o generar nuevas enfermedades o tener propósitos de destrucción de la humanidad, por ejemplo.

2.) Igualmente se considera fundamental que esta explotación de recursos se haga de mane- 
ra limpia y de tal manera que no vaya a producir una contaminación nociva ni cambios desfavorables en el medio ambiente terrestre y lunar, tal y como lo señala el artículo $9^{\text {no }}$ del Tratado del $67^{24}$ y el artículo $7^{\text {mo }}$ del Tratado de la Luna ${ }^{25}$. Con esto se busca no solo prevenir daños en el medio ambiente terrestre causados por la introducción en el de sustancias ajenas, sino brindar una protección ex ante al medio ambiente lunar, pues creemos que la humanidad tiene el deber de preservar la Luna como patrimonio cultural que es. Relacionado con lo anterior, creemos que el régimen de explotación a los recursos lunares debe atender al principio del desarrollo sostenible a fin de que las futuras generaciones puedan seguir teniendo acceso al tales recursos, esto de conformidad con lo establecido en el artículo $4^{\text {to }}$ del Tratado de la Luna, que señala que "en la exploración y utilización de la Luna se tendrán debidamente en cuenta los intereses de generaciones actuales y venideras" (Cursivas nuestras). Esto quiere decir que el tema de la explotación de los recursos de la Luna no puede llevar a su agotamiento y explotación desmedida sin medir las consecuencias de dicha explotación a futuro.
3.) Por otro lado, dado que el Tratado del 67, en su artículo primero, establece la libertad de exploración y utilización del espacio ultraterrestre, la Luna y otros cuerpos celestes, consideramos que los Estados en capacidad de mantener una explotación de recursos naturales en el espacio deben tener derecho a extraer y explotar los recursos en condiciones de igualdad, sin discriminación (evitando así que se cree un monopolio de explotación por parte de un solo Estado o compañía) y de manera responsable siempre que su actividad no interfiera con la actividad de otros Estados. Si tienen sospecha que su actividad pueda llegar a interferir con la de otros Estados deberán iniciar consultas. De esta manera se quiere establecer un régimen basado en la reciprocidad y la cooperación.

4.) Se ha considerado que dada una eventual explotación de recursos naturales extraterrestres, que sería una actividad con fines eminentemente comerciales (sin perjuicio que dichos recursos luego de ser comercializados podrían tener diversos fines), sería posible plantear un régimen similar al establecido por el principio $12^{\text {do }}$ de la Resolución de la ONU 41/65 de 1986 relativo a la teleobservación de la tierra ${ }^{26}$.

26 En el principio $12^{\text {do }}$ de dicha resolución se afirma el derecho que tienen los Estados objeto de la teleobservación a tener acceso a los datos primarios elaborados y obtenidos a partir de la teleobservación de su territorio: "Tan pronto que son producidos datos primarios y datos elaborados que correspondan al territorio bajo su jurisdicción, el estado objeto de la tele observación tendrá acceso a ellos sin discriminación, y a un costo razonable. Tendrá acceso, así mismo sin discriminación y en idénticas condiciones, teniendo particularmente en cuenta las necesidades e intereses de los países en desarrollo, a la información analizada disponible que corresponda al territorio bajo su jurisdicción y que posea cualquier estado que participe en actividades de tele observación. (Cursivas y énfasis nuestros). 
Basándose en esta idea, se propone un régimen jurídico que consagre el derecho que tienen todos los países del mundo, particularmente los países en desarrollo, a un acceso justo y equitativo, no solo a los beneficios que se deriven de esa explotación de recursos, sino a los recursos mismos. En efecto, todos los países del mundo deben tener derecho al acceso justo y equitativo a los recursos naturales de la Luna sin ningún tipo de discriminación, pero a un costo razonable que al mismo tiempo atienda las necesidades especiales de los países en desarrollo.

A través de esta disposición no solo habría un derecho como tal de todos los Estados sobre los recursos naturales del espacio ultraterrestre sino que, como contraprestación, los Estados que ejercieran tal explotación tendrían también derecho a recibir un precio razonable a cambio de su esfuerzo e inversión, logrando así un equilibrio entre dos intereses contrapuestos. Con este planteamiento, igualmente se tendría en cuenta lo dispuesto por el artículo 11.7 literal d) del Tratado de la Luna, en cuanto que el régimen contemplaría una participación equitativa de todos los Estados Parte en los beneficios obtenidos de esos recursos, teniendo especialmente en cuenta los intereses y necesidades de los países en desarrollo, así como los esfuerzos de los países que hayan contribuido directa o indirectamente a la explotación de la Luna.

5.) Se considera que el régimen internacional debe incluir la obligación de establecer una comisión o autoridad integrada por todos los Estados Parte de este régimen internacional, para hacer seguimientos constantes a la explotación y aprovechamiento de dichos recursos y que a su vez se encargue de guiar y reglamentar las particularidades de cómo se debe llevar a cabo la explotación de dichos recursos.

6.) Dado que se tiene el convencimiento que la actividad espacial en el futuro va estar concentrada principalmente en manos de privados o asociaciones entre privados por sus capacidades económicas superiores a la de las entidades gubernamentales, consideramos que es esencial que un régimen internacional para la explotación de recursos naturales extraterrestres contemple y regule con detalle la participación y rol de privados en dicha exploración, explotación y aprovechamiento de los recursos naturales de la Luna, teniendo siempre en cuenta los principios ya mencionados.

7.) Este régimen que se propone deberá adaptarse a las nuevas tecnologías y dinámicas del espacio, de modo que deberá tener en cuenta que las actividades de explotación de recursos naturales probablemente serán llevadas a cabo en colaboración con robots o vehículos espaciales controlados desde la Tierra y contemplar entre sus disposiciones dicha posibilidad.

Finalmente, se desea hacer énfasis en que una regulación más específica y detallada solo será posible cuando se tenga conocimiento sobre el fin de esos recursos naturales y las tecnologías disponibles en ese momento para su explotación. 


\section{CONCLUSIONES}

La Luna ha tenido una gran importancia cultural en la historia del ser humano y esta es una cuestión que a lo largo el trabajo se ha tratado de evidenciar. No solo desde los inicios de la civilización este cuerpo celeste ha formado parte del imaginario del hombre, sino que ha sido fuente de inspiración de múltiples creaciones culturales. Por esta razón, no puede ser objeto de ninguna clase de apropiación pues sería restar a la humanidad parte de su cultura y de su inspiración. Precisamente de ahí deriva la importancia de mantener la Luna como patrimonio común de la humanidad y de lograr que cada vez más Estados ratifiquen el Tratado de la Luna que plantea dicho principio.

La importancia política de la llegada a la Luna en el contexto de la Guerra Fría fue trascendental en el desarrollo de este conflicto ideológico, ya que significó una importante afirmación de poder tecnológico y de capacidad de desarrollo científico. Para los EE.UU. ser el primer Estado en llevar un hombre a la Luna, era la forma perfecta de ganar la carrera espacial luego de todas las victorias de la Unión Soviética en el espacio.

Ahora bien, en lo referente al ámbito legal es posible concluir que el Tratado de la Luna no ha recibido la atención suficiente en la comunidad internacional por referirse a temas que para muchos Estados resultan todavía una realidad lejana: la explotación de sus recursos naturales de la Luna u otros cuerpos celestes. La escasez de actividades en la Luna desde 1979 (que apenas hasta hace unos años resurge) y los pocos Estados Parte del mismo, contribuyen a lo anterior. Sin embargo, hay un elemento que ha quedado de la legislación existente y es que la Luna y los otros cuerpos celestes no pueden ser objeto de apropiación de ningún tipo: esto incluye reivindicaciones de propiedad por parte de particulares y organizaciones no gubernamentales así como de Estados y de entidades gubernamentales.

A su vez se puede afirmar que el elemento central del Acuerdo de la Luna, su mayor novedad y aporte, es el artículo XI relativo a los recursos naturales y al principio de patrimonio común de la humanidad para describir la Luna y sus recursos naturales. Según lo dispuesto en este artículo, la expresión patrimonio común de la humanidad no puede ser interpretada de manera aislada del resto del Tratado de la Luna. Esto ha llevado a concluir que, mientras no se cree un nuevo régimen internacional que rija la explotación de los recursos naturales en la Luna, éstos seguirán siendo considerados patrimonio común de la humanidad. No obstante, una eventual explotación de recursos naturales en la Luna implica una inversión multimillonaria (localizar, llegar efectivamente a extraer dichos recursos, el establecimiento de una base en la zona de explotación, transportar esos recursos a la tierra, etc.) la cual, mientras el estatus de los recursos naturales sea el de patrimonio común de la humanidad, ningún país está dispuesto a desembolsar. Si se aceptara, en un régimen internacional, la posibilidad de que dichos recursos puedan ser objeto de apropiación bajo ciertas condiciones (como el derecho de todos los países a tener acceso dichos recursos a un 
costo razonable y las demás condiciones mencionadas en la sección anterior de este artículo) esta explotación podría llegar a ser factible más pronto de lo esperado. No obstante, no deja de ser una objeción justificada a este último planteamiento la de los países que se podrían catalogar como "free-riders", quienes a pesar de no haber invertido capital propio, esfuerzo y mano de obra en lograr hacer viable la explotación de los recursos naturales tendrían derechos sobre los mismos. Podemos entonces considerar que el elemento más novedoso del Tratado de la Luna es que, a pesar de que propone el establecimiento de un futuro régimen de explotación sobre los recursos naturales, reafirma e insiste en el principio general de no apropiación de la Luna y otros cuerpos celestes, sin que esto niegue la posibilidad de que en un futuro puedan establecerse derechos de propiedad sobre los recursos naturales.

Hoy día la comunidad internacional no ha visto la importancia de establecer este régimen jurídico particular para la explotación de recursos naturales por la simple razón de que tal explotación no es factible en la actualidad, ya sea por razones económicas, el estado actual de la ciencia ${ }^{27} \mathrm{y} /$ o porque no se ha desarrollado una utilidad práctica para los mismos. Pero es evi-

27 Hasta antes del 2009, los científicos y geólogos del espacio especulaban que en los polos de la Luna podían existir millones toneladas de hidrogeno en la forma de agua congelada. Si bien las misiones y las muestras traídas por sondas espaciales en los años 90's que apoyaban esta idea- gracia a la presencia de hidrogeno-, no había certeza científica absoluta de la presencia de agua en los polos de la Luna. No fue sino hasta el 2009, que se logró confirmar la presencia de agua congelada en los cráteres del polo norte lunar gracias a la sonda Chandrayaan 1 la cual llevaba consigo un radar de la NASA diseñado para tal fin. Sin embargo falta mucho tiempo para que una explotación de dicho recurso llegue a ser viable. (Trinidad, K, 2010). dente que en el momento que la industria y/o ciencia logre establecer una verdadera utilidad para los recursos de la Luna y una forma de hacer dicha explotación económica y físicamente viable, habrá una gran auge de actividades de explotación de los recursos de la Luna y la necesidad establecer dicho régimen jurídico que gobierne esta actividad, será imperiosa.

Asimismo es importante anotar que el régimen jurídico actual relativo a la Luna tiene vacíos que en el futuro va a ser necesario completar. Por ejemplo, el Tratado no contempla que el futuro en la exploración y utilización los recursos de la Luna estén en manos de privados. Por esto es esencial que un régimen internacional al respecto contemple y regule en detalle la participación y el rol de privados en dicha exploración, explotación y aprovechamiento de los recursos naturales de la Luna.

\section{BIBLIOGRAFÍA}

10 películas sobre la luna. (s.f.). Canal total. Recuperado de http://www.canaltotal.com/10peliculas-sobre-la-luna/

Asamblea General de las Naciones Unidas. (1963). Declaración de los principios legales que deben regir las actividades de los Estados en la exploración y uso del espacio ultraterrestre. Aprobada por la Resolución 1962 (XVII), de 13 de diciembre de 1963. Recuperado de http://www.oosa.unvienna.org/pdf/ publications/STSPACE11S.pdf 
---. (1966). Tratado sobre los Principios que deben regir las Actividades de los Estados en la Exploración del Espacio Ultraterrestre, incluida la Luna y otros Cuerpos Celestes. Aprobado por la Resolución 2222 (XVI), de 19 de diciembre de 1966

--- (1979). Acuerdo que debe regir las actividades de los Estados en la Luna y otros cuerpos celestes. Aprobado el 5 de diciembre de 1979. Nueva York, NY. Recuperado de http://www.dipublico.com.ar/instrumentos/54.html

--. (1997). Declaración sobre la cooperación internacional en la exploración y utilización del espacio ultraterrestre en beneficio e interés de todos los Estados, teniendo especialmente en cuenta las necesidades de los países en desarrollo. Aprobada por la Resolución 51/122, de 4 de febrero de 1997. Recuperado de http://www.dipublico.com.ar/3991/ declaracion-sobre-la-cooperacion-internacional-en-la-exploracion-y-utilizacion-del-espacio-ultraterrestre-en-beneficio-e-interes-detodos-los-estados-teniendo-especialmenteen-cuenta-las-necesidades/

---. (2002). Tratados y principios de las Naciones Unidas sobre el Espacio Ultraterrestre. Organización de las Naciones Unidas. Nueva York, NY. Recuperado de http://www.oosa.unvienna.org/pdf/publications/STSPACE11S.pdf

--. (2011). Declaración con ocasión del quincuagésimo aniversario del primer vuelo espacial tripulado y del quincuagésimo aniversario de la Comisión sobre la Utilización del Espacio Ultraterrestre con Fines Pacíficos. Aprobada sobre la base del informe de la Comisión Política Especial y de Descolonización (Cuarta Comisión )(A/66/425 )el 9 de diciembre de 2011. Recuperado de http://daccess-ddsny.un.org/doc/UNDOC/GEN/N11/463/11/ PDF/N1146311.pdf?OpenElement

United Nations Committee on the Peaceful Uses of Outer Space, COPUOS. (2008).

Naciones Unidas. Asamblea General. "Resolución 3201 [Declaration on the Establishment of a New International Economic Order]". 1 mayo 1974. Recuperado de http://www.undocuments.net/s6r3201.ht

Artola, R. (2009). La Carrera Espacial: del Sputnik al Apollo 11. Madrid: Alianza Editorial.

Benkö, M. \& Schrogl, K. (2005). Essential Air and Space Law. The Nettherlands: Eleven International Publishing.

Brandt-Erichsen, D. (Noviembre-dicembre de 1994). The $L 5$ Society. Reimpreso de Ad Astra, the magazine of the National Space Society, Nov.-Dec., 1994. Recuperado de http:// www.nss.org/settlement/L5news/L5history. htm

Centro de información de las Naciones Unidas. (s.f.). Espacio ultraterrestre. Recuperado de http://www.cinu.mx/temas/derecho-internacional/espacio-ultraterrestre/ 
Cobo, B. (1990). Inca Religion and Customs. [1653]. Austin, Texas: University of Texas Press.

Cocca, A. (1964). Determination of the meaning of the expression "res communis hummanitatis". En Space Law. Proceeding of the Sixth Colloquium on the Law of Outer Space, Washington.

Crovisier, J. (2012). De la Terre à la Lune. [1865] et Autour de la Lune [1870]: les débuts de la conquête spatiale. Observatoire de ParisMeudon. Recuperado de http://www.lesia. obspm.fr/perso/jacques-crovisier/JV/verne_ TLAL.html

Demac, D. (1988). Tracing New Orbits-Cooperation and Competition in Global Satellite Development, 13-16. Nueva York. Columbia University Press.

Diederiks-Verschoor, I. \& Kopal V (Eds.). (2008). An Introduction to Space Law, (3 ${ }^{\text {ra }}$ Ed.). Países Bajos. Kluwer Law International. pp. 4852

Jasetunliyana. (Ed.), Space law: development and scope. pp. 2-14. Recuperado de http:// books.google.com.co/books?id=|209Gfn9A $5 \mathrm{IC} \&$ printsec $=$ frontcover $\& \mathrm{hl}=\mathrm{es} \&$ source $=\mathrm{g}$ bs_ge_summary_r\&cad $=0 \# v=$ onepage $\& q \& f$ $=$ false

Farrel, P., Neil-Jones, N. \&, Zubritsky, E. (30 de agosto de 2012). Walls of lunar crater may hold patchy ice, LRO radar finds. Lunar Re- connaissance Orbiter. Recuperado de http:// www.nasa.gov/mission_pages/LRO/news/ shackleton-ice.html

Ferrer. M. (1976). Derecho Espacial. Buenos Aires: Editorial Plus Extra.

Francoz, A. (1973). El régimen jurídico político de la Luna. Recuperado de http://biblio.juridicas.unam.mx/libros/4/1973/9.pdf

Gangale, T. (Enero 2008). Common Heritage in Magnificent Desolation, Conferencia para la $46^{\text {th }}$ Aerospace Sciences Meeting and Exhibit, American Institute of Aeronautics and Astronautics. AIAA. Reno, Nevada. Recuperado de http://www.astrosociology.com/library/ pdf/asm2008_magnificentdesolation.pdf

Grimal, P. (1986). The Dictionary of Classical Mythology. Oxford: Basil Blackwell.

---. (2009). The Development of Outer Space: Sovereignty and Property Rights in International Space Law. Santa Barbara: Praeger.

Google Lunar X Prize. (s.f.). Recuperado de http://www.googlelunarxprize.org

Guzmán. S. (1881). El Derecho de la Conquista y La Teoría del Equilibrio en la América Latina. Recuperado de http://archive.org/stream/ elderechodeconq00guzmgoog\#page/n17/ mode/2up

Hall, M. D. (1985). A Study of Sumerian Moon God Nanna/Suen. (Disertación doctoral), University of Pennsylvania. Recuperado de 
http://repository.upenn.edu/dissertations/ AAI8603645/

Harlan, G. (2003). Key Objections to the Moon Treaty. National Space Society Chapters Network. Recuperado de http://www.nsschapters.org/hub/pdf/MoonTreatyObjections.pdf

Hirsch, F. (1978). The Hollywood Epic. South Brunswick and New York. London: A.S. Barnes \& Co.

Hotakainen, M. (2008). Mars. From Myth to Mystery to Recent Discoveries. New York: Springer.

How china may be the next to land on the moon.

(27 de agosto de 2012). Agence France Presse. Recuperado de http://www.afp.com/en/ node/445482

International Astronomical Union. (s.f.). Naming of astronomical objects. Recuperado de http://www.iau.org/public/themes/naming/

--. (s.f.). Questions and answers on planets. Recuperado de http://www.iau.org/public_ press/news/release/iau0603/questions_ answers/

Jasentuliyana, N. (Ed.). (1984). Maintaining Outer Space for Peaceful Uses: Proceedings of a Symposium Held in the Hague, March 1984. United Nations University, Tokio, Japón.

Kopal, V. (2009). Tratado sobre los principios que deben regir las actividades de los Esta- dos en la exploración y utilización del espacio ultraterrestre, incluso la Luna y otros cuerpos celestes. Naciones Unidas. Recuperado de http://legal.un.org/avl/pdf/ha/tos/tos_s.pdf

Lachs, M. (1977). El derecho del espacio ultraterrestre. México D.F.: Fondo de Cultura Económica.

Laxman, S. (7 de marzo de 2012). Chang'e-3: China to launch first moon rover in 2013. Asian Scientist. Recuperado de http://www. asianscientist.com/topnews/chang-e-3-china-first-moon-rover-launch-in-2013/

Listner, M. (24 de octubre de 2011). The Moon Treaty: failed international law or waiting in the shadows? Space Review. Recuperado de http://www.thespacereview.com/article/1954/1

Lozano, A. (2007). La Guerra Fría. Barcelona: Melusina.

Lyall, F., \& Larsen P. (2009). The Moon and other Celestial Bodies. En Space Law a Treatise, 175-199. Recuperado de http://lawlib. shirazu.ac.ir:8080/pdfTemp/space\%20lawatreaty.pdf

Martos, A. (2009). Breve Historia de la Carrera Espacial. Madrid: Editorial Nowtilus.

Mejía, M. (2008). Propiedad privada y soberanía en el espacio. Biblioteca Jurídica Virtual del Instituto de Investigaciones Jurídicas de la UNAM, 171-206. Recuperado de http://biblio.juridicas.unam.mx/libros/6/2790/9.pdf 
Méliès, G. (Productor y Director). (1902). Le voyage dans la lune [Película]. Francia: Star. Recuperado de http://www.imdb.com/title/ tt0000417/

Moskowitz, C. (19 de octubre de 2012). Private Space Taxi Builders On Track to Launch Astronauts Soon. Space.com. Recuperado de http://www.space.com/18128-privatespace-taxis-nasa-progress.html\#sthash.KgOZ7ary.dpuf

Nasa reconoce que perdió piedras lunares y otras muestras del espacio. (9 de diciembre de 2011). Rpp noticas. Recuperado de http:// www.rpp.com.pe/2011-12-09-nasa-reconoce-queperdio-piedras-lunares-y-otras-muestras-del-espacionoticia_430340.html

National Aeronautics and Space Administration, NASA (2004). The vision for space exploration. Recuperado de http://www.nasa.gov/ pdf/55584_main_vision_space_explorationhi-res.pdf

---. (2012). The decision to go to the moon: President John F. Kenned'ys may 25, 1961 speech before a joint session of congress. History Office. Recuperado de http://history.nasa. gov/moondec.html

---. (2012). The Lagrange Points. Recuperado de http://map.gsfc.nasa.gov/mission/observatory_I2.html

---. (s.f.). Missions. Solar System Exploration. Recuperado de http://solarsystem.nasa.gov/ missions/index.cfm
Obama abandona el plan para el retorno del hombre a la Luna. (30 de enero de 2010). Corporación Radiotelevisión Española. Recuperado de http://www.rtve.es/noticias/20100130/obama-abandona-plan-para-retorno-del-hombre-luna/315120.shtml

Oficina de asuntos legales. (2013). United Nations Treaty Collection. Organización de las Naciones Unidas. Recuperado de http://treaties.un.org/Pages/ViewDetails.aspx?src=IND\&mtdsg_no=XXIV$2 \&$ chapter $=24 \&$ lang $=$ en

Oficina para asuntos del espacio exterior (UNOOSA). (s.f.). Treaty Database: Treaty signatures. Organización de las Naciones Unidas. Recuperado de http://www.oosa.unvienna. org/oosatdb/showTreatySignatures.do

---. (s.f.). International Space Law. Organización de las Naciones Unidas. Recuperado de http://www.unoosa.org/oosa/en/SpaceLaw/index.html.

Rodríguez, E. (2006). Nuestro derecho al espacio. La órbita geoestacionaria: ¿Una frustrada regulación? Elementos de Juicio. Revista de Temas Constitucionales, 2, 51-85. Recuperado de http://www.juridicas.unam.mx/ publica/librev/rev/juicio/cont/2/cnt/cnt4. pdfSagan, C. (1980). Cosmos. Nueva York: Random House

---. (1996). Un punto azul pálido. Una visión del futuro humano en el espacio. Barcelona: Editorial Planeta S.A. 
Sciences Meeting and Exhibit. Recuperado dehttp://www.astrosociology.com/Library/ PDF/ASM2008_MagnificentDesolation.pdf

Stone, W. (s.f.). Mining the Moon. Discovery news. Recuperado de http://dsc.discovery. com/space/features/mining-the-moon.html

Sharp. T. (19 de octubre de 2012). How big is the Moon? Space.com. Recuperado de http://www.space.com/18135-how-big-isthe-moon.html

Teitel, A. (7 de septiembre de 2012). Pillaging the moon for the promise of space energy. Discovery news. Recuperado de http://news. discovery.com/space/space-energy-miningthe-moon-120907.htm

Thode, R. (1997-2010). El Panteón egipcio: Jonsu. Recuperado de http://www.egiptologia. org/mitologia/panteon/jonsu.htm

Úbedad, J.E. (2011). Breve Introducción al derecho internacional Público. Madrid: Editorial Aeibus.

Trinidad, K. (1 de marzo de 2010). More Moon Ice. National Aeronautics and Space Administration. Recuperado de http://solar-
system.nasa.gov/news/display.cfm?News_ ID $=33595$

Wall, M. (12 de septiembre de 2011). Giant Leaps: Top milestones of human spaceflight: reaching for the stars. Space.com. Recuperado de http://www.space.com/11329-human-spaceflight-biggest-moments-50th-anniversary.html

---. (7 de noviembre de 2012). NASA May Unveil New Manned Moon Missions Soon. Space. com. Recuperado de http://www.space. com/18380-nasa-moon-missions-obamaelection.html?cid=dlvr.it

Zamarripa, N. (2001). Régimen legal internacional entorno al patrimonio común de la humanidad. Derecho y cultura, 4, 121-243 Recuperado de http://www.juridicas.unam. $\mathrm{mx} /$ publica/librev/rev/derycul/cont/4/ens/ ens13.pdf

Zak, A. (2008). Russia details its grand space strategy in 2010s New deep-space ships, big rockets and nuclear space tugs are promised at the Moscow air show. Rusian space web. Recuperado de http://www.russianspaceweb.com/spacecraft_manned_2010s. html 